\title{
The Effectiveness of Acupuncture for Dysphagia after Stroke: A Systematic Review and Meta-Analysis
}

\author{
Lida Zhong $\mathbb{D}^{1},{ }^{1}$ Jing Wang $\left(\mathbb{D},{ }^{1}\right.$ Fang Li $\mathbb{D}^{1},{ }^{1}$ Xiao Bao $\mathbb{D}^{1},{ }^{1}$ Huiyu Liu $\mathbb{D}^{1},{ }^{1}$ and Pu Wang ${ }^{2}$ \\ ${ }^{1}$ Department of Rehabilitation Medicine, Yuebei People's Hospital, Shaoguan, Guangdong 512026, China \\ ${ }^{2}$ Department of Rehabilitation Medicine, The Seventh Affiliated Hospital Sun Yat-sen University, Shenzhen, \\ Guangdong 518107, China
}

Correspondence should be addressed to Huiyu Liu; liuhuiyudoctor@sohu.com and Pu Wang; wangpu_03@126.com

Received 27 August 2020; Revised 10 October 2020; Accepted 7 January 2021; Published 19 January 2021

Academic Editor: Feng Zhang

Copyright (C) 2021 Lida Zhong et al. This is an open access article distributed under the Creative Commons Attribution License, which permits unrestricted use, distribution, and reproduction in any medium, provided the original work is properly cited.

\begin{abstract}
Objectives. This study reviewed and evaluated existing evidence of the efficacy of acupuncture as a clinical treatment for dysphagia after stroke. Methods. Five English and four Chinese databases were searched from inception to March 2020. All randomized controlled trials (RCTs) incorporating acupuncture or acupuncture combined with other interventions for the treatment of dysphagia after stroke were enrolled. All data were independently assessed and extracted by two authors. The bias risk assessment recommended by the Cochrane Collaboration's tool was used to assess the quality of the selected studies. This meta-analysis was conducted by using RevMan 5.3. Pooled analyses were calculated by the mean difference (MD) and 95\% confidence interval (CI). Heterogeneity was assessed by the $I^{2}$ test. Results. Thirty-five studies involving 3024 patients were analyzed. The meta-analysis showed that the therapeutic efficacy of acupuncture combined with other interventions was better than that of the control group for the standardized swallowing assessment (SSA) score (MD $=-3.78,95 \% \mathrm{CI}$ : -4.64 to $-2.91, P<0.00001$ ), Ichiro Fujishima rating scale (IFRS) score $(\mathrm{MD}=1.68,95 \% \mathrm{CI}$ : 1.16 to $2.20, P<0.00001)$, videofluoroscopic swallowing study (VFSS) score $(\mathrm{MD}=2.26,95 \% \mathrm{CI}: 1.77$ to $2.74, P<0.00001)$, and water swallowing test (WST) score $(\mathrm{MD}=-1.21,95 \% \mathrm{CI}:-1.85$ to -0.57 , $P=0.0002$ ). In studies reporting adverse effects, no serious outcome from an adverse event was confirmed. Conclusion. This systematic review indicated that acupuncture could be an effective therapy for treating dysphagia after stroke although stricter evaluation standards and rigorously designed RCTs are needed.
\end{abstract}

\section{Introduction}

Dysphagia is one of the most common poststroke sequelae, accounting for 27 to $64 \%$ of stroke patients [1], and is often associated with malnutrition, pneumonia, and dehydration [2]. The previous study [3] has shown that dysphagia after stroke affects quality of life, carries increased risks of mortality and dependency, prolongs hospital stays, increases healthcare costs, and often leads to discharge from the hospital to a care home. Therefore, to accelerate the recovery of swallowing function and reduce these risks, it is very important to find an effective treatment for dysphagia.

At present, there are many treatments for dysphagia, such as behavioral interventions, drug therapy, physical stimulation, and transcranial magnetic stimulation. Some of these treatments have made considerable progress [4]. However, clinical evidence to establish their roles in the management of poststroke dysphagia is limited [4], and there is no clear treatment for dysphagia.

Acupuncture, as a form of alternative medicine, is a traditional treatment that is clinically effective for neurological diseases $[5,6]$. Acupuncture treatment exerts therapeutic effects by inserting a needle at specific acupoints on the body surface with stimulation delivery via manual rotation or electric pulses [7-9]. Some randomized controlled trials (RCTs) $[10,11]$ have shown that acupuncture may reduce the proportion of participants with dysphagia at the end of the trial. However, despite the high heterogeneity, the latest updated Cochrane review [12] on swallowing therapy, which included an analysis of acupuncture, failed to show 
improvement in swallowing ability. There is still a lack of high-quality research on acupuncture treatment of dysphagia [12], and many clinical studies are still in the preliminary stage, with great differences in the acupuncture methods and the selection of acupoints in the research, leading to the inconclusive conclusion of acupuncture treatment for dysphagia.

This systematic review and meta-analysis aimed to evaluate the potential availability and safety of acupuncture for poststroke dysphagia.

\section{Methods}

The protocol was registered on the International Platform of Registered Systematic Review and Meta-analysis Protocols (INPLASY2020100036), and it was conducted according to the preferred reporting items for systematic reviews and meta-analysis (PRISMA): The PRISMA Statement [13].

2.1. Search Strategy. We searched the following databases from their inception until March 2020: EMBASE (via Ovid), MEDLINE (via Ovid), the Cochrane library (via Ovid), PubMed (via website), ScienceDirect (via website), China National Knowledge Infrastructure (CNKI) (via website), China Biology Medicine disc (CBMdisc) (via website), China Science and Technology Journal Database (VIP) (via website), and Wanfang Data (via website). Manual searches of relevant references were also conducted. The search terms were ("dysphagia," "swallowing disorders," "deglutition disorders," or "swallowing dysfunction") and ("stroke," "cerebral apoplexy," or "cerebrovascular accident") and ("acupuncture," "needling," "electroacupuncture," or "warm acupuncture").

\subsection{Inclusion and Exclusion Criteria}

2.2.1. Types of Studies. All RCTs of acupuncture for dysphagia after stroke were selected and excluded nonrandomized studies, observational studies, animal studies, qualitative studies, and letters.

2.2.2. Types of Participants. All patients conformed to the explicit clinical diagnosis criteria of stroke and dysphagia: (1) the participants were clinically diagnosed with ischemic or hemorrhagic stroke by computerized tomography or magnetic resonance imaging; (2) dysphagia was diagnosed using a clinical bedside swallowing assessment, a videofluoroscopic swallowing study (VFSS), or a fiberoptic endoscopic examination of swallowing (FEES).

2.2.3. Types of Interventions. For the intervention in experimental trials, acupuncture alone or acupuncture combined with other interventions was included, and other interventions included behavioral interventions, drug therapy, and electrical stimulation. The interventions should be the same between experimental and control trials, except for acupuncture in the experimental trials.
2.2.4. Types of Outcome Measures. The clinical symptoms had obviously improved with specific evaluation standards, such as (1) Watian swallowing test (WST) [14], (2) standardized swallowing assessment (SSA) [15-17], (3) penetration-aspiration scale (PAS) [18], and (4) functional oral intake scale (FOIS) [19], or by using an objective index, such as (1) VFSS [20] and (2) endoscopic evaluation of swallowing [21], as the efficacy evaluation criterion.

2.3. Data Extraction. Data were extracted by three review authors (Lida Zhong, Jing Wang, and Fang Li) independently using a standardized form after evaluation. Disagreements were resolved with the assistance from a fourth author (Pu Wang), if necessary. Data extracted included the surname of the first author, year of publication, intervention used in the acupuncture and control groups, evaluation time, outcomes, conclusions, follow-up duration, and adverse effects.

2.4. Risk of Bias Assessment. The included RCTs were assessed according to the Cochrane risk of bias assessment tool [22], and this process was carried out independently by the two review authors (Lida Zhong and Jing Wang). Quality was assessed as having a low, an unclear, or a high risk of bias according to seven criteria: (1) random allocation method (selection bias); (2) allocation concealment (selection bias); (3) blinding of assessors (performance bias); (4) blinding of outcome assessment (detection bias); (5) integrity of data results (attrition bias); (6) selective reporting (reporting bias); and (7) other sources of bias. Any disagreements that arouse at any stage between the two reviews were resolved through discussion with a third author (Pu Wang).

2.5. Statistical Analysis. All statistical analyses were performed using RevMan 5.3 (http://ims.cochrane.org/revman). For dichotomous variables, the relative risk (RR) with its $95 \%$ confidence interval (CI) was calculated. For continuous variables, the mean difference (MD) and standardized mean difference (SMD) with their 95\% CIs were calculated. The heterogeneity between each group was tested by Cochran's $Q$ statistic and the $I^{2}$ test [23]. Studies with an $I^{2}$ of $25 \%$ to $50 \%$ were considered to have low heterogeneity, and $I^{2}$ values of $50 \%$ to $75 \%$ and $>75 \%$ were considered indicative of moderate and high levels of heterogeneity, respectively. Fixed-effect models were used to combine studies if the $I^{2}$ test was not significant $(P$ for heterogeneity $<0.1)$. Otherwise, randomeffect models were used. $P<0.05$ was considered statistically significant for the between-group difference. If substantial heterogeneity was detected, we looked for reasonable explanations, and subgroup analysis or sensitivity analysis could be applied to explore the causes of heterogeneity. If the sources of heterogeneity could not be determined, a descriptive analysis was adopted.

\section{Results}

3.1. Study Selection. The PRISMA flow diagram of the literature search and the results are shown in Figure 1. These 


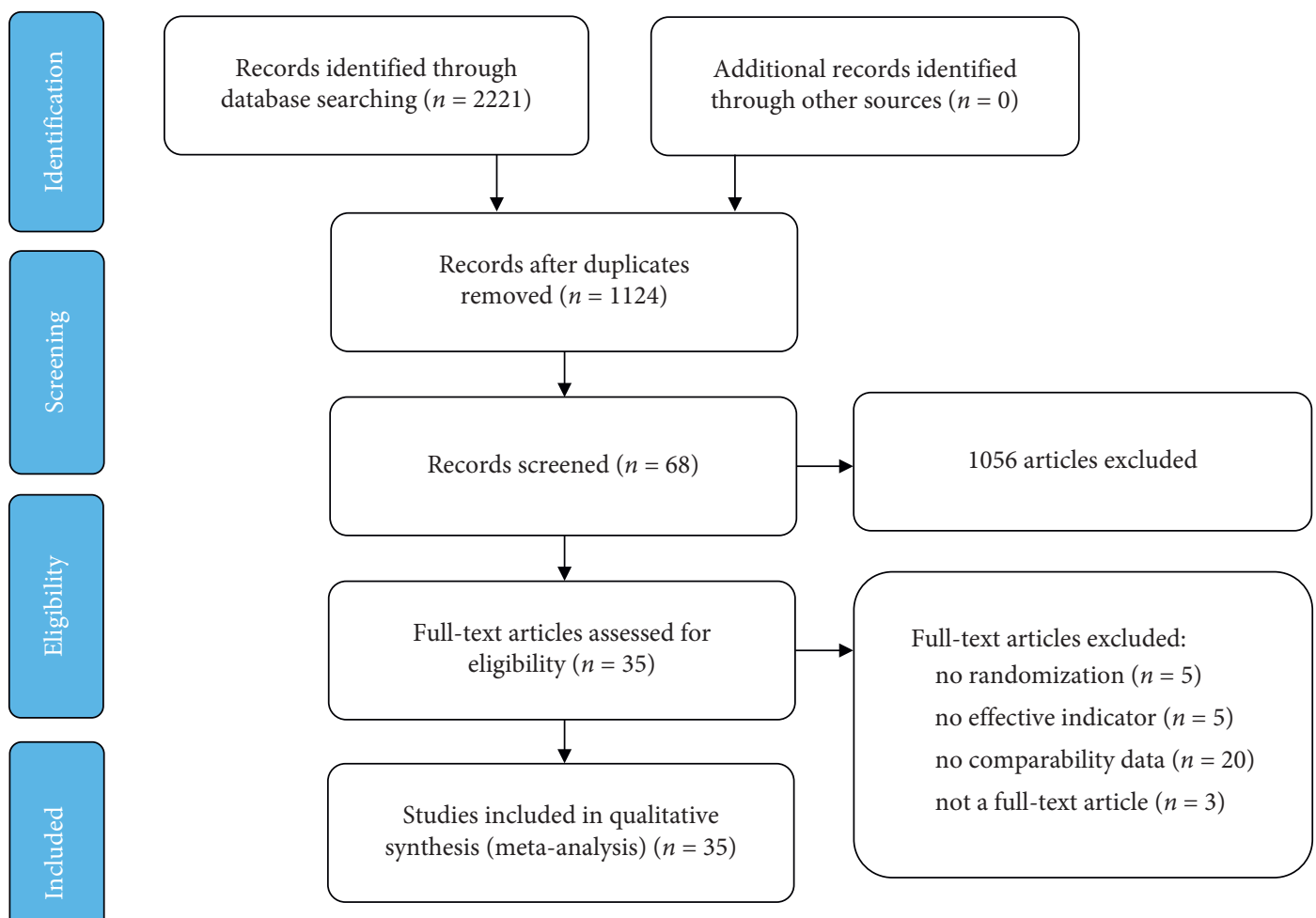

Figure 1: Flow diagram for the selection of the included studies.

studies were screened for eligibility using the detailed participant, intervention, comparison, and outcome (PICO) criteria. The initial search of computerized databases retrieved a total of 2221 articles. After removing duplicates, 1124 articles were found, of which 68 records were subjected to a full-text review. We excluded 33 articles for the following reasons: no randomization $(n=5)$, no effective indicator $(n=5)$, no comparability data $(n=20)$, and not a full-text article $(n=3)$. Finally, $35 \mathrm{RCTs}$ were included in this review.

3.2. Description of Studies. The characteristics of the included studies in this review are shown in Table 1. Among the 35 included studies, all the studies were conducted in China. Nine of thirty-five articles [24, 26-28, 31, 32, $35,38,41]$ were reported in an English database, and the remaining were reported in a Chinese database. Overall, 35 eligible studies involved 3024 participants diagnosed with dysphagia after stroke, and they were published between 2006 and 2020. All trials compared acupuncture with a swallowing treatment. In these trials, the frequency of acupuncture intervention was at least three times a week for more than two weeks in duration. Three studies [28, 32, 49] reported adverse events, and four studies [25, 28, 31, 40] reported dropouts.

3.3. Assessment for Risk of Bias. The details of the overall risk of bias across the 35 RCTs are provided in Table 2. Of the 35 included studies, the randomization procedure was reported in adequate detail in all studies. One trial [28] clearly reported the allocation concealment, the blinding of participants and personnel, and the blinding of outcome assessment; other descriptions in the other studies were unclear. Four trials $[25,28,31,40]$ excluded dropout participants for the data analysis, which may increase the risk of attrition bias. All the studies clearly described the selective reporting. In total, 4 out of 35 studies (11.43\%) were judged as having a high risk of bias because one of the main aspects of the bias assessments was high (Figures 2 and 3).

3.4. Standard Swallowing Assessment (SSA). There were 13 studies that used the SSA as the effective evaluation standard with continuous data. The meta-analysis showed a MD with high heterogeneity $\left(I^{2}=80 \%\right)$. Therefore, the random-effect model was used (Figure 4), and we performed a subgroup analysis according to the course of the disease. Heterogeneity was found to remain unaltered although no source for it was identified. Meanwhile, the meta-analysis results showed significant differences in SSA scores in dysphagia between the acupuncture and control groups. The acupuncture group had lower SSA scores than the control group $(\mathrm{MD}=-3.78, \quad 95 \% \quad \mathrm{CI}:-4.64$ to $-2.91, \quad P<0.00001)$ (Figure 4).

3.5. Ichiro Fujishima Rating Scale (IFRS). Twelve studies used the Ichiro Fujishima rating scale as the evaluation standard. The meta-analysis indicated that the acupuncture group had obviously improved IFRS scores ( $M D=1.68,95 \%$ 
TABLE 1: Characteristics of included studies.

\begin{tabular}{|c|c|c|c|c|c|}
\hline Reference & Participants & Intervention & Acupoints & Outcome measures & $\begin{array}{l}\text { Main } \\
\text { conclusion }\end{array}$ \\
\hline $\begin{array}{l}\text { Wang et al. } \\
{[24]}\end{array}$ & $\begin{array}{l}\text { G1 (50): } \\
62.14 \pm 12.14 \\
\text { G2 }(50): \\
62.37 \pm 5.34\end{array}$ & $\begin{array}{l}\text { G1: A + ST, G2: ST, F: } 3 \\
\text { times/week, D: } 4 \text { weeks }\end{array}$ & $\begin{array}{l}\text { GB20, GB12, BL10, RN23, ST5, } \\
\text { ST40, EX-HN12, EX-HNl3 }\end{array}$ & WST + IFRS & $\begin{array}{c}1 \text { WST } \\
(\mathrm{G} 1<\mathrm{G} 2) \\
2 \text { IFRS } \\
(\mathrm{G} 1>\mathrm{G} 2)\end{array}$ \\
\hline $\begin{array}{l}\text { Jiang et al. } \\
{[25]}\end{array}$ & $\begin{array}{c}\text { G1 (65): } \\
60 \pm 10 \\
\text { G2 (65): } \\
60 \pm 9\end{array}$ & $\begin{array}{l}\text { G1: A + ST + NEST, G2: } \\
\text { ST + NEST, F: } 5 \text { times/ } \\
\text { week, D: } 4 \text { weeks }\end{array}$ & Shesanzhen (extra) & HAMA + HAMD + SSA + sEMG & $\begin{array}{c}\mathrm{G} 1<\mathrm{G} 2 \text { in all } \\
\text { outcomes }\end{array}$ \\
\hline $\begin{array}{l}\text { Wu et al. } \\
{[26]}\end{array}$ & $\begin{array}{c}\text { G1 (65): } \\
44.00 \pm 2.92 \\
\text { G2 (63): } \\
44.35 \pm 2.60 \\
\text { G1 }(50):\end{array}$ & $\begin{array}{l}\text { G1: A + ST, G2: ST, F: } 5 \\
\text { times/week, D: } 6 \text { weeks }\end{array}$ & $\begin{array}{c}\text { EX-HN1, GV20, EX-HN5, GB20, } \\
\text { CV23 }\end{array}$ & IFRS & $\begin{array}{c}\text { IFRS } \\
(\mathrm{G} 1>\mathrm{G} 2)\end{array}$ \\
\hline $\begin{array}{l}\text { Wei et al. } \\
\text { (2015) [27] }\end{array}$ & $\begin{array}{c}61.50 \pm 4.2 \\
\mathrm{G} 2(50): \\
62.50 \pm 4.90\end{array}$ & $\begin{array}{l}\text { G1: A + ST, G2: ST, F: } 7 \\
\text { times/week, D: } 2 \text { weeks }\end{array}$ & $\begin{array}{l}\text { RN23, RN22, EX-HN12, EX- } \\
\text { HN13, LI4, PC6, ST36 }\end{array}$ & VFSS + MBI + FIM & $\begin{array}{c}\mathrm{G} 1>\mathrm{G} 2 \text { in all } \\
\text { outcomes }\end{array}$ \\
\hline $\begin{array}{l}\text { Xia et al. } \\
{[28]}\end{array}$ & $\begin{array}{l}\text { G1 }(67): 67 \pm 9 \\
\text { G2 (63): } \\
\quad 66 \pm 10\end{array}$ & $\begin{array}{l}\text { G1: A + ST, G2: ST, F: } 6 \\
\text { times/week, D: } 6 \text { weeks }\end{array}$ & $\begin{array}{c}\text { PC6, DU26, SP6, HT1, LU5, } \\
\text { BL40, GV20, GB20, CV23, } \\
\text { Jialianquan (extra), EX-HN12, } \\
\text { EX-HN13 }\end{array}$ & VFSS + SSA + BI + SWAL-QOL & $\begin{array}{c}1 \text { SSA } \\
(\mathrm{G} 1<\mathrm{G} 2) \\
2 \mathrm{G} 1>\mathrm{G} 2 \text { in } \\
\text { other } \\
\text { outcomes }\end{array}$ \\
\hline $\begin{array}{l}\text { Zeng et al. } \\
{[29]}\end{array}$ & $\begin{array}{c}\text { G1 }(25): \\
58.01 \pm 10.74 \\
\text { G2 }(25): \\
57.98 \pm 11.82\end{array}$ & $\begin{array}{l}\text { G1: A + ST, G2: ST, F: } 7 \\
\text { times/week, D: } 4 \text { weeks }\end{array}$ & $\begin{array}{l}\text { SJ17, GB12, Ex-HN14, SI17, } \\
\text { RN22, ST9, Toupizhen (extra), } \\
\text { RN23, EX-HN12, EX-HN13, } \\
\text { Tunyan (extra), Tiyan (extra), } \\
\text { ST4, ST6, DU26, RN24 }\end{array}$ & IFRS & $\begin{array}{c}\text { IFRS } \\
(\mathrm{G} 1>\mathrm{G} 2)\end{array}$ \\
\hline $\begin{array}{l}\text { Chang } \\
\text { et al. [30] }\end{array}$ & $\begin{array}{l}\text { G1 }(38): \\
46 \pm 10 \\
\text { G2 }(36): \\
44 \pm 11\end{array}$ & $\begin{array}{c}\text { G1: A + BT + NEST, } \\
\text { G2: BT + NEST, F: } 6 \\
\text { times/week, D: } 4 \text { weeks }\end{array}$ & $\begin{array}{l}\text { GV20, RN23, EX-HN12, EX- } \\
\text { HN13, Toupizhen (extra) }\end{array}$ & IFRS & $\begin{array}{c}\text { IFRS } \\
(\mathrm{G} 1>\mathrm{G} 2)\end{array}$ \\
\hline $\begin{array}{l}\text { Chen et al. } \\
{[31]}\end{array}$ & $\begin{array}{l}\text { G1 }(50): \\
67 \pm 11 \\
\text { G2 }(50) \\
67 \pm 10\end{array}$ & $\begin{array}{l}\text { G1: A + ST, G2: ST, F: } 6 \\
\text { times/week, D: } 8 \text { weeks }\end{array}$ & $\begin{array}{c}\text { GB20, Ex-HN14, Gongxue } \\
\text { (extra), Zhiqiang (extra), Tunyan } \\
\text { (extra), Fayin (extra), RN23, EX- } \\
\text { HN12, EX-HN13 }\end{array}$ & RSST + WST + SSA & $\begin{array}{c}1 \text { WST } \\
(\mathrm{G} 1<\mathrm{G} 2) \\
2 \text { RSST } \\
(\mathrm{G} 1>\mathrm{G} 2) \\
3 \mathrm{SSA} \\
(\mathrm{G} 1<\mathrm{G} 2)\end{array}$ \\
\hline $\begin{array}{l}\text { Feng et al. } \\
\text { [32] }\end{array}$ & $\begin{array}{l}\text { G1 } \\
60 \pm 12 \\
\text { G2 }(30): \\
58 \pm 12\end{array}$ & $\begin{array}{l}\text { G1: A + ST, G2: ST, F: } 7 \\
\text { times/week, D:3 weeks }\end{array}$ & $\begin{array}{c}\text { RN23, SJ17, GB12, GB20, DU16, } \\
\text { DU15, ST5, EX-HN12, EX- } \\
\text { HN13, Shezhen (extra) }\end{array}$ & VFSS + WST & $\begin{array}{l}1 \text { WST } \\
(\mathrm{G} 1<\mathrm{G} 2) \\
2 \text { VFSS } \\
(\mathrm{G} 1>\mathrm{G} 2) \\
1 \text { WST }\end{array}$ \\
\hline $\begin{array}{l}\text { Guo et al. } \\
{[33]}\end{array}$ & $\begin{array}{l}\text { G1 }(50): \\
66.21 \pm 8.03 \\
\text { G2 }(50): \\
65.91 \pm 7.85\end{array}$ & $\begin{array}{l}\text { G1: A + ST, G2: ST, F: } 6 \\
\text { times/week, D: } 4 \text { weeks }\end{array}$ & $\begin{array}{l}\text { DU15, DU16, BL10, Zhiqiang } \\
\text { (extra), RN23 }\end{array}$ & $\mathrm{SSA}+\mathrm{WST}+\mathrm{BI}$ & $\begin{array}{c}1 \text { WST } \\
(\mathrm{G} 1<\mathrm{G} 2) \\
2 \mathrm{BI} \\
(\mathrm{G} 1>\mathrm{G} 2) \\
3 \mathrm{SSA} \\
(\mathrm{G} 1<\mathrm{G} 2)\end{array}$ \\
\hline $\begin{array}{l}\text { He et al. } \\
{[34]}\end{array}$ & $\begin{array}{l}\text { G1 }(60): \\
62.16 \pm 7.04 \\
\text { G2 }(60): \\
61.83 \pm 6.81\end{array}$ & $\begin{array}{l}\text { G1: A + ST, G2: ST, F: } 5 \\
\text { times/week, D: } 8 \text { weeks }\end{array}$ & GB20, SJ17, RN23 & $\mathrm{SSA}+\mathrm{WST}+\mathrm{BI}$ & $\begin{array}{c}1 \text { WST } \\
(\mathrm{G} 1<\mathrm{G} 2) \\
2 \mathrm{BI} \\
(\mathrm{G} 1>\mathrm{G} 2) \\
3 \mathrm{SSA} \\
(\mathrm{G} 1<\mathrm{G} 2)\end{array}$ \\
\hline $\begin{array}{l}\text { Li et al. } \\
{[35]}\end{array}$ & $\begin{array}{l}\text { G1 }(42): \\
57.4 \pm 4.8 \\
\text { G2 }(42): \\
57.4 \pm 4.8\end{array}$ & $\begin{array}{c}\mathrm{G} 1: \mathrm{A}+\mathrm{BT}+\mathrm{ST}, \mathrm{G} 2: \\
\mathrm{BT}+\mathrm{ST}, \mathrm{F}: 6 \text { times/ } \\
\text { week, D: } 4 \text { weeks }\end{array}$ & Shezhen (extra) & VFSS & $\begin{array}{c}\text { VFSS } \\
(\mathrm{G} 1>\mathrm{G} 2)\end{array}$ \\
\hline
\end{tabular}


TABle 1: Continued.

\begin{tabular}{|c|c|c|c|c|c|}
\hline Reference & Participants & Intervention & Acupoints & Outcome measures & $\begin{array}{c}\text { Main } \\
\text { conclusion }\end{array}$ \\
\hline $\begin{array}{l}\text { Li et al. } \\
\text { [36] }\end{array}$ & $\begin{array}{l}\text { G1 (50): } \\
42.6 \pm 2.3 \\
\text { G2 (50): } \\
42.5 \pm 2.2\end{array}$ & $\begin{array}{l}\text { G1: A + ST, G2: ST, F: } 7 \\
\text { times/week, D: } 3 \text { weeks }\end{array}$ & $\begin{array}{c}\text { GB20, Ex-HN14, Gongxue } \\
\text { (extra), Zhiqiang (extra), Tunyan } \\
\text { (extra) RN23, EX-HN12, EX- } \\
\text { HN13 }\end{array}$ & IFRS + WST & $\begin{array}{c}1 \text { WST } \\
(\mathrm{G} 1<\mathrm{G} 2) \\
2 \text { IFRS } \\
(\mathrm{G} 1>\mathrm{G} 2)\end{array}$ \\
\hline $\begin{array}{l}\mathrm{Li} \text { and } \mathrm{Gu} \\
{[37]}\end{array}$ & $\begin{array}{l}\text { G1 }(40): \\
61.9 \pm 7.9 \\
\text { G2 }(40): \\
63.6 \pm 6.9\end{array}$ & $\begin{array}{c}\mathrm{G} 1: \mathrm{A}+\mathrm{BT}+\mathrm{ST}, \mathrm{G} 2: \\
\mathrm{BT}+\mathrm{ST}, \mathrm{F}: 6 \text { times/ } \\
\text { week, D: } 4 \text { weeks }\end{array}$ & GB20, DU16, HT5, LR3 & PAS + SSA + WST & $\begin{array}{c}\mathrm{G} 1<\mathrm{G} 2 \text { in all } \\
\text { outcomes }\end{array}$ \\
\hline $\begin{array}{l}\text { Liu et al. } \\
\text { [38] }\end{array}$ & $\begin{array}{l}\text { G1 }(36): \\
57.6 \pm 8.2 \\
\text { G2 }(36): \\
58.5 \pm 8.7\end{array}$ & $\begin{array}{l}\text { G1: A + BT, G2: BT, F: } 6 \\
\text { times/week, D: } 8 \text { weeks }\end{array}$ & $\begin{array}{c}\text { GB20, Ex-HN14, CV23, Ex- } \\
\text { HN12, Ex-HN13, SP6, LR3, } \\
\text { ST40, LI4 }\end{array}$ & WST & $\begin{array}{c}\text { WST } \\
(\mathrm{G} 1<\mathrm{G} 2)\end{array}$ \\
\hline $\begin{array}{l}\text { Qiao et al. } \\
\text { [39] }\end{array}$ & $\begin{array}{c}\text { G1 }(43): \\
52.27 \pm 10.45 \\
\text { G2 }(43): \\
52.86 \pm 10.72\end{array}$ & $\begin{array}{c}\mathrm{G} 1: \mathrm{A}+\mathrm{BT}+\mathrm{ST}, \mathrm{G} 2: \\
\mathrm{BT}+\mathrm{ST}, \mathrm{F}: 3 \text { times/ } \\
\text { week, D: } 2 \text { weeks }\end{array}$ & $\begin{array}{l}\text { GB20, DU16, EX-HN15, RN23, } \\
\text { Jialianquan (extra), EX-HN12, } \\
\text { EX-HN13, LI4, LR3, HT5 }\end{array}$ & IFRS + WST & $\begin{array}{l}1 \text { WST } \\
(\mathrm{G} 1<2) \\
2 \text { IFRS } \\
(\mathrm{G} 1>\mathrm{G} 2)\end{array}$ \\
\hline $\begin{array}{l}\text { Wang et al. } \\
{[40]}\end{array}$ & $\begin{array}{l}\text { G1 }(35): \\
64+8 \\
\text { G2 }(35): \\
65 \pm 9\end{array}$ & $\begin{array}{l}\text { G1: A + ST, G2: ST, F: } 5 \\
\text { times/week, D: } 3 \text { weeks }\end{array}$ & Shesanzhen (extra) & SSA + WST + SWAL-QOL & $\begin{array}{c}1 \text { WST } \\
(\mathrm{G} 1<\mathrm{G} 2) \\
2 \text { SWAL- } \\
\text { QOL } \\
(\mathrm{G} 1>\mathrm{G} 2) \\
3 \mathrm{SSA} \\
(\mathrm{G} 1<\mathrm{G} 2)\end{array}$ \\
\hline Wang [41] & $\begin{array}{l}\text { G1 }(30): \\
57.6 \pm 9.1 \\
\text { G2 }(30): \\
59.6 \pm 8.9\end{array}$ & $\begin{array}{c}\text { G1: A + BT + NEST, } \\
\text { G2: BT + NEST, F: } 7 \\
\text { times/week, D: } 2 \text { weeks }\end{array}$ & $\begin{array}{c}\text { RN23, Jialianquan (extra), HT5, } \\
\text { DU20, Zuqianjin (extra) } \\
\text { Zuwujin (extra) }\end{array}$ & WST & $\begin{array}{c}\text { WST } \\
(\mathrm{G} 1<\mathrm{G} 2)\end{array}$ \\
\hline $\mathrm{Xu}[42]$ & $\begin{array}{l}\text { G1 }(38): \\
62.74 \pm 5.19 \\
\text { G2 }(38): \\
63.19 \pm 4.38\end{array}$ & $\begin{array}{l}\text { G1: A + BT, G2: BT, F: } 5 \\
\text { times/week, D: } 4 \text { weeks }\end{array}$ & $\begin{array}{l}\text { GB20, BL10, DU16, RN23, EX- } \\
\text { HN12, EX-HN13, PC6, HT5, SP6, } \\
\text { ST36, RN12, BL23, SP3, KI3 }\end{array}$ & WST & $\begin{array}{c}\text { WST } \\
(\mathrm{G} 1<\mathrm{G} 2)\end{array}$ \\
\hline $\begin{array}{l}\text { Yang et al. } \\
{[43]}\end{array}$ & $\begin{array}{c}\text { G1 (30): } 65.8 \\
\text { G2 (30): } 67.3 \\
\text { G1 (40): }\end{array}$ & $\begin{array}{l}\text { G1: A + BT, G2: BT, F: } 5 \\
\text { times/week, D: } 2 \text { weeks }\end{array}$ & GV20, SJ17, RN23, SP6, ST36 & WST & $\begin{array}{c}\text { WST } \\
(\mathrm{G} 1<\mathrm{G} 2) \\
1 \text { WST }\end{array}$ \\
\hline $\begin{array}{l}\text { Yu et al. } \\
{[44]}\end{array}$ & $\begin{array}{c}63 \pm 10 \\
\mathrm{G} 2(38) \\
64 \pm 11\end{array}$ & $\begin{array}{l}\text { G1: A + ST, G2: ST, F: } 7 \\
\text { times/week, D: } 3 \text { weeks }\end{array}$ & $\begin{array}{l}\text { DU26, LI4, DU15, DU16, GB20, } \\
\text { RN23 }\end{array}$ & IFRS + WST & $\begin{array}{l}(\mathrm{G} 1<\mathrm{G} 2) \\
2 \text { IFRS } \\
(\mathrm{G} 1>\mathrm{G} 2)\end{array}$ \\
\hline Zhu [45] & $\begin{array}{c}\text { G1 }(50): \\
65.05 \pm 8.99 \\
\text { G2 }(48): \\
64.03 \pm 9.83\end{array}$ & $\begin{array}{c}\text { G1: A + BT + ST, G2: } \\
\text { BT + ST, F: } 6 \text { times/ } \\
\text { week, D: } 4 \text { weeks }\end{array}$ & $\begin{array}{c}\text { GB20, GB12, SJ17, } \\
\text { Shanglianquan (extra), ST9 }\end{array}$ & SSA + SWAL-QOL & $\begin{array}{c}1 \text { SWAL- } \\
\text { QOL } \\
(\mathrm{G} 1>\mathrm{G} 2) \\
2 \text { SSA } \\
(\mathrm{G} 1<\mathrm{G} 2)\end{array}$ \\
\hline $\begin{array}{l}\text { Zhou et al. } \\
\text { [46] }\end{array}$ & $\begin{array}{c}\text { G1 (34): } \\
59.90 \pm 3.87 \\
\text { G2 (34): } \\
60.43 \pm 4.07\end{array}$ & $\begin{array}{c}\text { G1: A + ST + NEST, G2: } \\
\text { ST + NEST, F: } 6 \text { times/ } \\
\text { week, D: } 4 \text { weeks }\end{array}$ & Toupizhen (extra) & $\mathrm{SSA}+\mathrm{SWAL}-\mathrm{QOL}+\mathrm{CT7R}$ & $\begin{array}{c}1 \text { SSA } \\
(\mathrm{G} 1<\mathrm{G} 2) \\
2 \mathrm{G} 1>\mathrm{G} 2 \text { in } \\
\text { other } \\
\text { outcomes }\end{array}$ \\
\hline $\begin{array}{l}\text { Zhou et al. } \\
\text { [47] }\end{array}$ & $\begin{array}{c}\text { G1 }(30): \\
68.30 \pm 13.84 \\
\text { G2 }(30): \\
70.26 \pm 11.97\end{array}$ & $\begin{array}{c}\text { G1: A + ST + NEST, G2: } \\
\text { ST + NEST, F: } 6 \text { times/ } \\
\text { week, D: } 2 \text { weeks }\end{array}$ & Toupizhen (extra) & $\mathrm{SSA}+\mathrm{VFSS}+\mathrm{WST}+\mathrm{CT} 7 \mathrm{R}$ & $\begin{array}{c}1 \text { CT7R } \\
(\mathrm{G} 1>\mathrm{G} 2) \\
2 \text { VFSS } \\
(\mathrm{G} 1>\mathrm{G} 2) \\
3 \mathrm{SSA} \\
(\mathrm{G} 1<\mathrm{G} 2) \\
4 \mathrm{WST} \\
(\mathrm{G} 1<\mathrm{G} 2)\end{array}$ \\
\hline
\end{tabular}


TABLE 1: Continued.

\begin{tabular}{|c|c|c|c|c|c|}
\hline Reference & Participants & Intervention & Acupoints & Outcome measures & $\begin{array}{c}\text { Main } \\
\text { conclusion }\end{array}$ \\
\hline $\begin{array}{l}\text { Zhi et al. } \\
\text { [48] }\end{array}$ & $\begin{array}{l}\text { G1 (39): } \\
63.16 \pm 6.92 \\
\text { G2 (39): } \\
62.78 \pm 6.78\end{array}$ & $\begin{array}{c}\mathrm{G} 1: \mathrm{A}+\mathrm{BT}+\mathrm{ST}, \mathrm{G} 2: \\
\mathrm{BT}+\mathrm{ST}, \mathrm{F}: 6 \text { times/ } \\
\text { week, D: } 12 \text { weeks }\end{array}$ & $\begin{array}{c}\text { RN23, Jialianquan (extra), EX- } \\
\text { HN12, EX-HN13, Shexiaxue } \\
\text { (extra) }\end{array}$ & $\mathrm{WST}+\mathrm{GUSS}+\mathrm{BI}+\mathrm{FMA}$ & $\begin{array}{c}1 \text { WST } \\
(\mathrm{G} 1<\mathrm{G} 2) \\
2 \mathrm{G} 1>\mathrm{G} 2 \text { in } \\
\text { other } \\
\text { outcomes } \\
1 \text { WST }\end{array}$ \\
\hline $\begin{array}{l}\text { Zhang and } \\
\mathrm{Li}[49]\end{array}$ & $\begin{array}{l}\text { G1 }(46): \\
66.2 \pm 7.4 \\
\text { G2 }(46): \\
67.5 \pm 6.7\end{array}$ & $\begin{array}{c}\text { G1: A + ST + NEST, G2: } \\
\text { ST + NEST, F: } 7 \text { times/ } \\
\text { week, D: } 4 \text { weeks }\end{array}$ & $\begin{array}{l}\text { RN23, Tunyan (extra), } \\
\text { Toupizhen (extra) }\end{array}$ & VFSS + WST + IFRS & $\begin{array}{c}(\mathrm{G} 1<\mathrm{G} 2) \\
2 \text { VFSS } \\
(\mathrm{G} 1>\mathrm{G} 2) \\
3 \text { IFRS } \\
(\mathrm{G} 1>\mathrm{G} 2)\end{array}$ \\
\hline $\begin{array}{l}\text { Zhang et al. } \\
\text { [50] }\end{array}$ & $\begin{array}{c}\text { G1 (19): } \\
64.10 \pm 8.20 \\
\text { G2 (18): } \\
65.58 \pm 10.64\end{array}$ & $\begin{array}{l}\text { G1: A + rTMS + BT, G2: } \\
\text { rTMS + BT, F: } 6 \text { times/ } \\
\text { week, D: } 4 \text { weeks }\end{array}$ & $\begin{array}{c}\text { DU20, EX-HN1, ST8, DU16, } \\
\text { GB20, RN23, Jialianquan (extra), } \\
\text { ST4, ST6, ST7 }\end{array}$ & MBSImP + OTT & $\begin{array}{c}\mathrm{G} 1<\mathrm{G} 2 \text { in all } \\
\text { outcomes }\end{array}$ \\
\hline $\begin{array}{l}\text { Zhang et al. } \\
\text { [51] }\end{array}$ & $\begin{array}{c}\text { G1 }(87): \\
64.61 \pm 9.70 \\
\text { G2 }(87): \\
63.86 \pm 10.55\end{array}$ & $\begin{array}{l}\text { G1: A + ST, G2: ST, F: } 3 \\
\text { times/week, D: } 8 \text { weeks }\end{array}$ & $\begin{array}{l}\text { DU16, GB20, RN23, Jialianquan } \\
\text { (extra), EX-HN15, EX-HN12, } \\
\text { EX-HN13, HT5, LR3, LI4 }\end{array}$ & IFRS + WST & $\begin{array}{c}1 \text { WST } \\
(\mathrm{G} 1<\mathrm{G} 2) \\
2 \text { IFRS } \\
(\mathrm{G} 1>\mathrm{G} 2)\end{array}$ \\
\hline $\begin{array}{l}\text { Zhang and } \\
\text { Yin [52] }\end{array}$ & $\begin{array}{c}\text { G1 (62): } \\
70 \pm 1 \\
\text { G2 (56): } \\
68 \pm 2\end{array}$ & $\begin{array}{l}\text { G1: A + ST, G2: ST, F: } 5 \\
\text { times/week, D: } 4 \text { weeks }\end{array}$ & Shenguan (extra), KI3, LR3 & $\mathrm{SSA}+\mathrm{WST}+\mathrm{IFRS}$ & $\begin{array}{c}1 \text { WST } \\
(\mathrm{G} 1<\mathrm{G} 2) \\
2 \text { SSA } \\
(\mathrm{G} 1<\mathrm{G} 2) \\
3 \text { IFRS } \\
(\mathrm{G} 1>\mathrm{G} 2)\end{array}$ \\
\hline $\begin{array}{l}\text { Zhang et al. } \\
\text { [53] }\end{array}$ & $\begin{array}{l}\text { G1 }(20): \\
58.3 \pm 10.1 \\
\text { G2 }(20): \\
58.2 \pm 10.1\end{array}$ & $\begin{array}{l}\text { G1: A + ST + NEST, G2: } \\
\text { ST + NEST, F: } 5 \text { times/ } \\
\text { week, D: } 4 \text { weeks }\end{array}$ & $\begin{array}{l}\text { Tunyan (extra), RN23, DU16, } \\
\text { SJ17, EX-HN12, EX-HNl3 }\end{array}$ & VFSS + sEMG & $\begin{array}{l}1 \text { VFSS } \\
(\mathrm{G} 1>\mathrm{G} 2) \\
2 \mathrm{sEMG} \\
(\mathrm{G} 1<\mathrm{G} 2)\end{array}$ \\
\hline $\begin{array}{l}\text { Yin et al. } \\
{[54]}\end{array}$ & $\begin{array}{c}\text { G1 (18): } \\
69.52 \pm 6.01 \\
\text { G2 (20): } \\
65.41 \pm 7.01\end{array}$ & $\begin{array}{l}\text { G1: A + ST + NEST, G2: } \\
\text { ST + NEST, F: } 5 \text { times/ } \\
\text { week, D: } 3 \text { weeks }\end{array}$ & $\begin{array}{c}\text { ST9, RN22, RN23, EX-HN12, } \\
\text { EX-HN13 }\end{array}$ & IFRS + WST & $\begin{array}{c}1 \text { WST } \\
(\mathrm{G} 1<\mathrm{G} 2) \\
2 \text { IFRS } \\
(\mathrm{G} 1>\mathrm{G} 2) \\
1 \text { VFSS }\end{array}$ \\
\hline $\begin{array}{l}\text { Gao et al. } \\
{[55]}\end{array}$ & $\begin{array}{l}\text { G1 }(30): \\
64 \pm 5 \\
\text { G2 }(30): \\
65 \pm 5\end{array}$ & $\begin{array}{c}\mathrm{G} 1: \mathrm{A}+\mathrm{BT}+\mathrm{ST}, \mathrm{G} 2: \\
\mathrm{BT}+\mathrm{ST}, \mathrm{F}: 5 \text { times/ } \\
\text { week, D: } 4 \text { weeks }\end{array}$ & $\begin{array}{c}\text { DU16, BL10, GB12, RN23, } \\
\text { Jialianquan (extra), EX-HN12, } \\
\text { EX-HNl3 }\end{array}$ & $\mathrm{VFSS}+\mathrm{SSA}+\mathrm{sEMG}$ & $\begin{array}{c}1 \text { VFSS } \\
(\mathrm{G} 1>\mathrm{G} 2) \\
2 \text { sEMG } \\
(\mathrm{G} 1<\mathrm{G} 2) \\
3 \mathrm{SSA} \\
(\mathrm{G} 1<\mathrm{G} 2)\end{array}$ \\
\hline Dong [56] & $\begin{array}{l}\text { G1 }(60): \\
55.3 \pm 6.4 \\
\text { G2 }(60): \\
55.3 \pm 6.4\end{array}$ & $\begin{array}{l}\text { G1: A + ST, G2: ST, F: } 5 \\
\text { times/week, D: } 2 \text { weeks }\end{array}$ & $\begin{array}{c}\text { EX-HN12, EX-HNl3, DU16, } \\
\text { DU15, RN23 }\end{array}$ & VFSS + WST & $\begin{array}{l}1 \text { WST } \\
(\mathrm{G} 1<\mathrm{G} 2) \\
2 \text { VFSS } \\
(\mathrm{G} 1>\mathrm{G} 2)\end{array}$ \\
\hline $\begin{array}{l}\text { Deng et al. } \\
\text { [57] }\end{array}$ & $\begin{array}{l}\text { G1 }(53): \\
59.2 \pm 11.6 \\
\text { G2 }(52): \\
59.8 \pm 13.2\end{array}$ & $\begin{array}{c}\text { G1: A + ST + NEST, G2: } \\
\text { ST + NEST, F: } 5 \text { times/ } \\
\text { week, D: } 3 \text { weeks }\end{array}$ & $\begin{array}{l}\text { PC6, DU26, SP6, GB20, SJ17, } \\
\text { GB12, Yanhoubi (extra), RN23 }\end{array}$ & WST + SSA & $\begin{array}{c}1 \text { WST } \\
(\mathrm{G} 1<\mathrm{G} 2) \\
2 \mathrm{SSA} \\
(\mathrm{G} 1<\mathrm{G} 2)\end{array}$ \\
\hline $\begin{array}{l}\text { Zhu et al. } \\
{[58]}\end{array}$ & $\begin{array}{l}\text { G1 (35): } \\
54.97 \pm 5.10 \\
\text { G2 }(35): \\
56.26 \pm 6.17\end{array}$ & $\begin{array}{c}\mathrm{G} 1: \mathrm{A}+\mathrm{BT}+\mathrm{ST}, \mathrm{G} 2: \\
\mathrm{BT}+\mathrm{ST}, \mathrm{F}: 6 \text { times/ } \\
\text { week, D: } 2 \text { weeks }\end{array}$ & $\begin{array}{l}\text { Yushizhen (extra), LI15, LI11, } \\
\text { LI10, SJ5, SJ3, LI4, ST32, GB34, } \\
\text { ST36, ST40, GB40, LR3, SP6 }\end{array}$ & IFRS + WST & $\begin{array}{l}1 \text { WST } \\
(\mathrm{G} 1<\mathrm{G} 2) \\
2 \text { IFRS } \\
(\mathrm{G} 1>\mathrm{G} 2)\end{array}$ \\
\hline
\end{tabular}

$\mathrm{G} 1>\mathrm{G} 2 / \mathrm{G} 1<\mathrm{G} 2$ indicates that the difference between the two groups was statistically significant, $P<0.05 ; \mathrm{G} 1=\mathrm{G} 2$ indicates that no significant differences were noted between the two groups, $P \geq 0.05$. G: group; G1: experimental group; G2: control group; A: acupuncture; ST: swallowing treatment; BT: basic treatment; NEST: neuromuscular electrical stimulation; rTMS: repetitive transcranial magnetic stimulation; F: frequency; D: duration; WST: Watian swallowing test; SSA: standard swallowing assessment; VFSS: videofluoroscopic swallowing study; IFRS: Ichiro Fujishima rating scale; SWAL-QOL: swallow quality-of-life questionnaire; BI: Barthel index; FMA: Fugl-Meyer assessment; CT7R: Caiteng 7 rank; sEMG: surface electromyography; HAMA: Hamilton anxiety scale; HAMD: Hamilton depression scale; RSST: repetitive saliva swallowing test; MBSImP: modified barium swallow impairment profile; OTT: oral transit time. 
TABLE 2: The risk of bias assessment.

\begin{tabular}{|c|c|c|c|c|c|c|}
\hline Reference & Randomization & $\begin{array}{c}\text { Allocation } \\
\text { concealment }\end{array}$ & Blinding & Incomplete data & $\begin{array}{c}\text { Selective } \\
\text { report }\end{array}$ & $\begin{array}{c}\text { Other } \\
\text { bias }\end{array}$ \\
\hline $\begin{array}{l}\text { Wang et al. } \\
{[24]}\end{array}$ & $\begin{array}{c}\text { Low risk } \\
\text { Randomized by random } \\
\text { number table }\end{array}$ & $\begin{array}{l}\text { Unclear risk } \\
\text { Allocation schedule } \\
\text { was not mentioned }\end{array}$ & $\begin{array}{l}\text { Unclear risk } \\
\text { Blinding unclear }\end{array}$ & $\begin{array}{l}\text { Low risk } \\
\text { None lost to } \\
\text { follow-up }\end{array}$ & $\begin{array}{l}\text { Low risk } \\
\text { All outcomes } \\
\text { reported }\end{array}$ & $\begin{array}{l}\text { Unclear } \\
\text { risk }\end{array}$ \\
\hline $\begin{array}{l}\text { Jiang et al. } \\
\text { [25] }\end{array}$ & $\begin{array}{c}\text { Low risk } \\
\text { Randomized by random } \\
\text { number table }\end{array}$ & $\begin{array}{l}\text { Unclear risk } \\
\text { Allocation schedule } \\
\text { was not mentioned }\end{array}$ & $\begin{array}{l}\text { Unclear risk } \\
\text { Blinding unclear }\end{array}$ & $\begin{array}{l}\text { High risk } \\
10 \text { participants } \\
\text { dropout }\end{array}$ & $\begin{array}{l}\text { Low risk } \\
\text { All outcomes } \\
\text { reported }\end{array}$ & $\begin{array}{l}\text { Unclear } \\
\text { risk }\end{array}$ \\
\hline Wu et al. [26] & $\begin{array}{c}\text { Low risk } \\
\text { Randomized by random } \\
\text { number table }\end{array}$ & $\begin{array}{l}\text { Unclear risk } \\
\text { Allocation schedule } \\
\text { was not mentioned }\end{array}$ & $\begin{array}{l}\text { Unclear risk } \\
\text { Blinding unclear }\end{array}$ & $\begin{array}{l}\text { Low risk } \\
\text { None lost to } \\
\text { follow-up }\end{array}$ & $\begin{array}{l}\text { Low risk } \\
\text { All outcomes } \\
\text { reported }\end{array}$ & $\begin{array}{l}\text { Unclear } \\
\text { risk }\end{array}$ \\
\hline $\begin{array}{l}\text { Wei et al. } \\
{[27]}\end{array}$ & $\begin{array}{c}\text { Low risk } \\
\text { Randomized by random } \\
\text { number table }\end{array}$ & $\begin{array}{l}\text { Unclear risk } \\
\text { Allocation schedule } \\
\text { was not mentioned }\end{array}$ & $\begin{array}{l}\text { Unclear risk } \\
\text { Blinding unclear }\end{array}$ & $\begin{array}{l}\text { Low risk } \\
\text { None lost to } \\
\text { follow-up }\end{array}$ & $\begin{array}{l}\text { Low risk } \\
\text { All outcomes } \\
\text { reported }\end{array}$ & $\begin{array}{l}\text { Unclear } \\
\text { risk }\end{array}$ \\
\hline Xia et al. [28] & $\begin{array}{c}\text { Low risk } \\
\text { Randomized by random } \\
\text { number table }\end{array}$ & $\begin{array}{c}\text { Low risk } \\
\text { Automated } \\
\text { assignment system }\end{array}$ & $\begin{array}{c}\text { Low risk } \\
\text { Participants and } \\
\text { outcome assessors } \\
\text { blinded }\end{array}$ & $\begin{array}{l}\text { High risk } \\
14 \text { participants } \\
\text { dropout }\end{array}$ & $\begin{array}{l}\text { Low risk } \\
\text { All outcomes } \\
\text { reported }\end{array}$ & $\begin{array}{l}\text { Unclear } \\
\text { risk }\end{array}$ \\
\hline $\begin{array}{l}\text { Zeng et al. } \\
\text { [29] }\end{array}$ & $\begin{array}{c}\text { Low risk } \\
\text { Randomized by random } \\
\text { number table }\end{array}$ & $\begin{array}{r}\text { Uncle } \\
\text { Allocatior } \\
\text { was not } \mathrm{r}\end{array}$ & $\begin{array}{l}\text { Unclear risk } \\
\text { Blinding unclear }\end{array}$ & $\begin{array}{r}\text { Lo } \\
\text { Non } \\
\text { foll }\end{array}$ & $\begin{array}{l}\text { Lov } \\
\text { All ov } \\
\text { rep }\end{array}$ & $\begin{array}{l}\text { Unclear } \\
\text { risk }\end{array}$ \\
\hline $\begin{array}{l}\text { Chang et al. } \\
{[30]}\end{array}$ & $\begin{array}{c}\text { Low risk } \\
\text { Randomized by random } \\
\text { number table. }\end{array}$ & $\begin{array}{l}\text { Unclear risk } \\
\text { Allocation schedule } \\
\text { was not mentioned }\end{array}$ & $\begin{array}{l}\text { Unclear risk } \\
\text { Blinding unclear }\end{array}$ & $\begin{array}{r}\text { Lo } \\
\text { Non } \\
\text { foll }\end{array}$ & $\begin{array}{l}\text { Loy } \\
\text { All ou } \\
\text { rep }\end{array}$ & $\begin{array}{l}\text { Unclear } \\
\text { risk }\end{array}$ \\
\hline $\begin{array}{l}\text { Chen et al. } \\
{[31]}\end{array}$ & $\begin{array}{c}\text { Low risk } \\
\text { Randomized by random } \\
\text { number table }\end{array}$ & $\begin{array}{l}\text { Unc } \\
\text { Allocatic } \\
\text { was not }\end{array}$ & $\begin{array}{c}\text { Unclear risk } \\
\text { Blinding unclear }\end{array}$ & $\begin{array}{r}\mathrm{Hi} \\
3 \mathrm{par} \\
\mathrm{dr}\end{array}$ & $\begin{array}{r}\text { Lo } \\
\text { All o } \\
\text { rel }\end{array}$ & $\begin{array}{l}\text { Unclear } \\
\text { risk }\end{array}$ \\
\hline $\begin{array}{l}\text { Feng et al. } \\
{[32]}\end{array}$ & $\begin{array}{c}\text { Low risk } \\
\text { Randomized by random } \\
\text { number table }\end{array}$ & $\begin{array}{l}\text { Uncle } \\
\text { Allocatio } \\
\text { was not }\end{array}$ & $\begin{array}{l}\text { Unclear risk } \\
\text { Blinding unclear }\end{array}$ & $\begin{array}{r}\text { Lo } \\
\text { Non } \\
\text { foll }\end{array}$ & $\begin{array}{r}\text { Lo } \\
\text { All o } \\
\text { rep }\end{array}$ & $\begin{array}{l}\text { Unclear } \\
\text { risk }\end{array}$ \\
\hline $\begin{array}{l}\text { Guo et al. } \\
{[33]}\end{array}$ & $\begin{array}{c}\text { Low risk } \\
\text { Randomized by random } \\
\text { number table }\end{array}$ & $\begin{array}{l}\text { Uncl } \\
\text { Allocatio } \\
\text { was not }\end{array}$ & $\begin{array}{l}\text { Unclear risk } \\
\text { Blinding unclear }\end{array}$ & $\begin{array}{r}\text { Lo } \\
\text { Non } \\
\text { foll }\end{array}$ & $\begin{array}{r}\text { Lo } \\
\text { All o } \\
\text { re }\end{array}$ & $\begin{array}{c}\text { Unclear } \\
\text { risk }\end{array}$ \\
\hline He et al. [34] & $\begin{array}{r}\text { Low } \\
\text { Randomizec } \\
\text { numbe }\end{array}$ & $\begin{array}{l}\text { Uncl } \\
\text { Allocatic } \\
\text { was not }\end{array}$ & $\begin{array}{l}\text { Unclear risk } \\
\text { Blinding unclear }\end{array}$ & $\begin{array}{r}\text { Lo } \\
\text { Non } \\
\text { fol }\end{array}$ & $\begin{array}{r}\text { Lov } \\
\text { All ov } \\
\text { rep }\end{array}$ & $\begin{array}{l}\text { Unclear } \\
\text { risk }\end{array}$ \\
\hline Li et al. [35] & $\begin{array}{r}\text { Low } \\
\text { Randomized } \\
\text { number }\end{array}$ & $\begin{array}{r}\text { Uncl } \\
\text { Allocatic } \\
\text { was not }\end{array}$ & $\begin{array}{l}\text { Unclear risk } \\
\text { Blinding unclear }\end{array}$ & $\begin{array}{r}\text { Lo } \\
\text { None } \\
\text { foll }\end{array}$ & $\begin{array}{l}\text { Lov } \\
\text { All ov } \\
\text { rep }\end{array}$ & $\begin{array}{l}\text { Unclear } \\
\text { risk }\end{array}$ \\
\hline Li et al. [36] & $\begin{array}{c}\text { Low risk } \\
\text { Randomized by random } \\
\text { number table }\end{array}$ & $\begin{array}{r}\text { Unc } \\
\text { Allocati } \\
\text { was not }\end{array}$ & $\begin{array}{l}\text { Unclear risk } \\
\text { Blinding unclear }\end{array}$ & $\begin{array}{r}\text { Lo } \\
\text { Non } \\
\text { foll }\end{array}$ & $\begin{array}{l}\text { Lor } \\
\text { All o } \\
\text { rep }\end{array}$ & $\begin{array}{l}\text { Unclear } \\
\text { risk }\end{array}$ \\
\hline $\begin{array}{l}\mathrm{Li} \text { and } \mathrm{Gu} \\
\text { [37] }\end{array}$ & $\begin{array}{c}\text { Low risk } \\
\text { Randomized by random } \\
\text { number table }\end{array}$ & $\begin{array}{l}\text { Unclear risk } \\
\text { Allocation schedule } \\
\text { was not mentioned }\end{array}$ & $\begin{array}{l}\text { Unclear risk } \\
\text { Blinding unclear }\end{array}$ & $\begin{array}{r}\text { Lo } \\
\text { Non } \\
\text { foll }\end{array}$ & $\begin{array}{r}\text { Lo } \\
\text { All o } \\
\text { rel }\end{array}$ & $\begin{array}{l}\text { Unclear } \\
\text { risk }\end{array}$ \\
\hline Liu et al. [38] & $\begin{array}{c}\text { Low risk } \\
\text { Randomized by random } \\
\text { number table }\end{array}$ & $\begin{array}{l}\text { Uncl } \\
\text { Allocatic } \\
\text { was not }\end{array}$ & $\begin{array}{l}\text { Unclear risk } \\
\text { Blinding unclear }\end{array}$ & $\begin{array}{l}\text { Low risk } \\
\text { None lost to } \\
\text { follow-up }\end{array}$ & $\begin{array}{r}\text { Lo } \\
\text { All o } \\
\text { re }\end{array}$ & $\begin{array}{l}\text { Unclear } \\
\text { risk }\end{array}$ \\
\hline $\begin{array}{l}\text { Qiao et al. } \\
\text { [39] }\end{array}$ & $\begin{array}{c}\text { Low risk } \\
\text { Randomized by random } \\
\text { number table }\end{array}$ & $\begin{array}{l}\text { Unclear risk } \\
\text { Allocation schedule } \\
\text { was not mentioned }\end{array}$ & $\begin{array}{l}\text { Unclear risk } \\
\text { Blinding unclear }\end{array}$ & $\begin{array}{l}\text { Low risk } \\
\text { None lost to } \\
\text { follow-up }\end{array}$ & $\begin{array}{l}\text { Low risk } \\
\text { All outcomes } \\
\text { reported }\end{array}$ & $\begin{array}{l}\text { Unclear } \\
\text { risk }\end{array}$ \\
\hline $\begin{array}{l}\text { Wang et al. } \\
{[40]}\end{array}$ & $\begin{array}{c}\text { Low risk } \\
\text { Randomized by random } \\
\text { number table }\end{array}$ & $\begin{array}{l}\text { Unclear risk } \\
\text { Allocation schedule } \\
\text { was not mentioned }\end{array}$ & $\begin{array}{l}\text { Unclear risk } \\
\text { Blinding unclear }\end{array}$ & $\begin{array}{l}\text { High risk } \\
8 \text { participants } \\
\text { dropout }\end{array}$ & $\begin{array}{l}\text { Low risk } \\
\text { All outcomes } \\
\text { reported }\end{array}$ & $\begin{array}{c}\text { Unclear } \\
\text { risk }\end{array}$ \\
\hline Wang [41] & $\begin{array}{c}\text { Low risk } \\
\text { Randomized by random } \\
\text { number table }\end{array}$ & $\begin{array}{l}\text { Unclear risk } \\
\text { Allocation schedule } \\
\text { was not mentioned }\end{array}$ & $\begin{array}{l}\text { Unclear risk } \\
\text { Blinding unclear }\end{array}$ & $\begin{array}{l}\text { Low risk } \\
\text { None lost to } \\
\text { follow-up }\end{array}$ & $\begin{array}{l}\text { Low risk } \\
\text { All outcomes } \\
\text { reported }\end{array}$ & $\begin{array}{c}\text { Unclear } \\
\text { risk }\end{array}$ \\
\hline $\mathrm{Xu}[42]$ & $\begin{array}{c}\text { Low risk } \\
\text { Randomized by random } \\
\text { number table }\end{array}$ & $\begin{array}{l}\text { Unclear risk } \\
\text { Allocation schedule } \\
\text { was not mentioned }\end{array}$ & $\begin{array}{l}\text { Unclear risk } \\
\text { Blinding unclear }\end{array}$ & $\begin{array}{l}\text { Low risk } \\
\text { None lost to } \\
\text { follow-up }\end{array}$ & $\begin{array}{l}\text { Low risk } \\
\text { All outcomes } \\
\text { reported }\end{array}$ & $\begin{array}{l}\text { Unclear } \\
\text { risk }\end{array}$ \\
\hline $\begin{array}{l}\text { Yang } \\
{[43]}\end{array}$ & $\begin{array}{c}\text { Low risk. } \\
\text { Randomized by random } \\
\text { number table. }\end{array}$ & $\begin{array}{l}\text { Unclear risk. } \\
\text { Allocation schedule } \\
\text { was not mentioned. }\end{array}$ & $\begin{array}{l}\text { Unclear risk. } \\
\text { Blinding unclear. }\end{array}$ & $\begin{array}{l}\text { Low risk. } \\
\text { None lost to } \\
\text { follow-up }\end{array}$ & $\begin{array}{l}\text { Low risk. } \\
\text { All outcomes } \\
\text { reported }\end{array}$ & $\begin{array}{c}\text { Unclear } \\
\text { risk }\end{array}$ \\
\hline
\end{tabular}


TABle 2: Continued.

\begin{tabular}{|c|c|c|c|c|c|c|}
\hline Reference & Randomization & $\begin{array}{c}\text { Allocation } \\
\text { concealment }\end{array}$ & Blinding & Incomplete data & $\begin{array}{l}\text { Selective } \\
\text { report }\end{array}$ & $\begin{array}{l}\text { Other } \\
\text { bias }\end{array}$ \\
\hline Yu et al. [44] & $\begin{array}{c}\text { Low risk } \\
\text { Randomized by random } \\
\text { number table }\end{array}$ & $\begin{array}{c}\text { Unclear risk } \\
\text { Allocation schedule } \\
\text { was not mentioned }\end{array}$ & $\begin{array}{c}\text { Unclear risk } \\
\text { Blinding unclear }\end{array}$ & $\begin{array}{l}\text { Low risk } \\
\text { None lost to } \\
\text { follow-up }\end{array}$ & $\begin{array}{l}\text { Low risk } \\
\text { All outcomes } \\
\text { reported }\end{array}$ & $\begin{array}{l}\text { Unclear } \\
\text { risk }\end{array}$ \\
\hline Zhu [45] & $\begin{array}{c}\text { Low risk } \\
\text { Randomized by random } \\
\text { number table }\end{array}$ & $\begin{array}{c}\text { Unclear risk } \\
\text { Allocation schedule } \\
\text { was not mentioned }\end{array}$ & $\begin{array}{l}\text { Unclear risk } \\
\text { Blinding unclear }\end{array}$ & $\begin{array}{l}\text { Low risk } \\
\text { None lost to } \\
\text { follow-up }\end{array}$ & $\begin{array}{l}\text { Low risk } \\
\text { All outcomes } \\
\text { reported }\end{array}$ & $\begin{array}{l}\text { Unclear } \\
\text { risk }\end{array}$ \\
\hline $\begin{array}{l}\text { Zhou et al. } \\
{[46]}\end{array}$ & $\begin{array}{c}\text { Low risk } \\
\text { Randomized by random } \\
\text { number table }\end{array}$ & $\begin{array}{l}\text { Unclear risk } \\
\text { Allocation schedule } \\
\text { was not mentioned }\end{array}$ & $\begin{array}{l}\text { Unclear risk } \\
\text { Blinding unclear }\end{array}$ & $\begin{array}{l}\text { Low risk } \\
\text { None lost to } \\
\text { follow-up }\end{array}$ & $\begin{array}{l}\text { Low risk } \\
\text { All outcomes } \\
\text { reported }\end{array}$ & $\begin{array}{l}\text { Unclear } \\
\text { risk }\end{array}$ \\
\hline $\begin{array}{l}\text { Zhou et al. } \\
\text { [47] }\end{array}$ & $\begin{array}{c}\text { Low risk } \\
\text { Randomized by random } \\
\text { number table }\end{array}$ & $\begin{array}{c}\text { Unclear risk } \\
\text { Allocation schedule } \\
\text { was not mentioned }\end{array}$ & $\begin{array}{l}\text { Unclear risk } \\
\text { Blinding unclear }\end{array}$ & $\begin{array}{l}\text { Low risk } \\
\text { None lost to } \\
\text { follow-up }\end{array}$ & $\begin{array}{l}\text { Low risk } \\
\text { All outcomes } \\
\text { reported }\end{array}$ & $\begin{array}{l}\text { Unclear } \\
\text { risk }\end{array}$ \\
\hline Zhi et al. [48] & $\begin{array}{c}\text { Low risk } \\
\text { Randomized by random } \\
\text { number table }\end{array}$ & $\begin{array}{l}\text { Unclea } \\
\text { Allocation } \\
\text { was not } \mathrm{m}\end{array}$ & $\begin{array}{l}\text { Unclear risk } \\
\text { Blinding unclear }\end{array}$ & $\begin{array}{l}\text { Low risk } \\
\text { None lost to } \\
\text { follow-up }\end{array}$ & $\begin{array}{l}\text { Low } \\
\text { All outc } \\
\text { repor }\end{array}$ & $\begin{array}{c}\text { Unclear } \\
\text { risk }\end{array}$ \\
\hline $\begin{array}{l}\text { Zhang and Li } \\
\text { [49] }\end{array}$ & $\begin{array}{c}\text { Low risk } \\
\text { Randomized by random } \\
\text { number table }\end{array}$ & $\begin{array}{l}\text { Uncle } \\
\text { Allocatio } \\
\text { was not } 1\end{array}$ & $\begin{array}{l}\text { Unclear risk } \\
\text { Blinding unclear }\end{array}$ & $\begin{array}{r}\text { Lo } \\
\text { Non } \\
\text { foll }\end{array}$ & $\begin{array}{r}\text { Lo } \\
\text { All o } \\
\text { rep }\end{array}$ & $\begin{array}{c}\text { Unclear } \\
\text { risk }\end{array}$ \\
\hline $\begin{array}{l}\text { Zhang et al. } \\
{[50]}\end{array}$ & $\begin{array}{c}\text { Low risk } \\
\text { Randomized by random } \\
\text { number table }\end{array}$ & $\begin{array}{c}\text { Unclear risk } \\
\text { Allocation schedule } \\
\text { was not mentioned }\end{array}$ & $\begin{array}{l}\text { Unclear risk } \\
\text { Blinding unclear }\end{array}$ & $\begin{array}{l}\text { Low risk } \\
\text { None lost to } \\
\text { follow-up }\end{array}$ & $\begin{array}{l}\text { Loy } \\
\text { All ou } \\
\text { rep }\end{array}$ & $\begin{array}{l}\text { Unclear } \\
\text { risk }\end{array}$ \\
\hline $\begin{array}{l}\text { Zhang et al. } \\
{[51]}\end{array}$ & $\begin{array}{c}\text { Low risk } \\
\text { Randomized by random } \\
\text { number table }\end{array}$ & $\begin{array}{l}\text { Unclear risk } \\
\text { Allocation schedule } \\
\text { was not mentioned }\end{array}$ & $\begin{array}{l}\text { Unclear risk } \\
\text { Blinding unclear }\end{array}$ & $\begin{array}{l}\text { Low risk } \\
\text { None lost to } \\
\text { follow-up }\end{array}$ & $\begin{array}{l}\text { Low risk } \\
\text { All outcomes } \\
\text { reported }\end{array}$ & $\begin{array}{l}\text { Unclear } \\
\text { risk }\end{array}$ \\
\hline $\begin{array}{l}\text { Zhang and } \\
\text { Yin [52] }\end{array}$ & $\begin{array}{c}\text { Low risk } \\
\text { Randomized by random } \\
\text { number table }\end{array}$ & $\begin{array}{l}\text { Unclear risk } \\
\text { Allocation schedule } \\
\text { was not mentioned }\end{array}$ & $\begin{array}{l}\text { Unclear risk } \\
\text { Blinding unclear }\end{array}$ & $\begin{array}{l}\text { Lo } \\
\text { None } \\
\text { foll }\end{array}$ & $\begin{array}{l}\text { Low risk } \\
\text { All outcomes } \\
\text { reported }\end{array}$ & $\begin{array}{c}\text { Unclear } \\
\text { risk }\end{array}$ \\
\hline $\begin{array}{l}\text { Zhang et al. } \\
{[53]}\end{array}$ & $\begin{array}{c}\text { Low risk } \\
\text { Randomized by random } \\
\text { number table }\end{array}$ & $\begin{array}{l}\text { Unclear risk } \\
\text { Allocation schedule } \\
\text { was not mentioned }\end{array}$ & $\begin{array}{l}\text { Unclear risk } \\
\text { Blinding unclear }\end{array}$ & $\begin{array}{l}\text { Low risk } \\
\text { None lost to } \\
\text { follow-up }\end{array}$ & $\begin{array}{l}\text { Low risk } \\
\text { All outcomes } \\
\text { reported }\end{array}$ & $\begin{array}{c}\text { Unclear } \\
\text { risk }\end{array}$ \\
\hline Yin et al. [54] & $\begin{array}{c}\text { Low risk } \\
\text { Randomized by random } \\
\text { number table }\end{array}$ & $\begin{array}{c}\text { Unclear risk } \\
\text { Allocation schedule } \\
\text { was not mentioned }\end{array}$ & $\begin{array}{l}\text { Unclear risk } \\
\text { Blinding unclear }\end{array}$ & $\begin{array}{l}\text { Low risk } \\
\text { None lost to } \\
\text { follow-up }\end{array}$ & $\begin{array}{l}\text { Low risk } \\
\text { All outcomes } \\
\text { reported }\end{array}$ & $\begin{array}{l}\text { Unclear } \\
\text { risk }\end{array}$ \\
\hline $\begin{array}{l}\text { Gao et al. } \\
{[55]}\end{array}$ & $\begin{array}{c}\text { Low risk } \\
\text { Randomized by random } \\
\text { number table }\end{array}$ & $\begin{array}{c}\text { Unclear risk } \\
\text { Allocation schedule } \\
\text { was not mentioned. }\end{array}$ & $\begin{array}{l}\text { Unclear risk } \\
\text { Blinding unclear }\end{array}$ & $\begin{array}{l}\text { Low risk } \\
\text { None lost to } \\
\text { follow-up }\end{array}$ & $\begin{array}{l}\text { Low risk } \\
\text { All outcomes } \\
\text { reported }\end{array}$ & $\begin{array}{l}\text { Unclear } \\
\text { risk }\end{array}$ \\
\hline Dong [56] & $\begin{array}{c}\text { Low risk } \\
\text { Randomized by random } \\
\text { number table }\end{array}$ & $\begin{array}{l}\text { Unclear risk } \\
\text { Allocation schedule } \\
\text { was not mentioned }\end{array}$ & $\begin{array}{l}\text { Unclear risk } \\
\text { Blinding unclear }\end{array}$ & $\begin{array}{l}\text { Low risk } \\
\text { None lost to } \\
\text { follow-up }\end{array}$ & $\begin{array}{l}\text { Low risk } \\
\text { All outcomes } \\
\text { reported }\end{array}$ & $\begin{array}{l}\text { Unclear } \\
\text { risk }\end{array}$ \\
\hline $\begin{array}{l}\text { Deng et al. } \\
\text { [57] }\end{array}$ & $\begin{array}{c}\text { Low risk } \\
\text { Randomized by random } \\
\text { number table }\end{array}$ & $\begin{array}{l}\text { Unclear risk } \\
\text { Allocation schedule } \\
\text { was not mentioned }\end{array}$ & $\begin{array}{l}\text { Unclear risk } \\
\text { Blinding unclear }\end{array}$ & $\begin{array}{l}\text { Low risk } \\
\text { None lost to } \\
\text { follow-up }\end{array}$ & $\begin{array}{l}\text { Low risk } \\
\text { All outcomes } \\
\text { reported }\end{array}$ & $\begin{array}{c}\text { Unclear } \\
\text { risk }\end{array}$ \\
\hline $\begin{array}{l}\text { Zhu et al. } \\
\text { [58] }\end{array}$ & $\begin{array}{c}\text { Low risk } \\
\text { Randomized by random } \\
\text { number table }\end{array}$ & $\begin{array}{l}\text { Unclear risk } \\
\text { Allocation schedule } \\
\text { was not mentioned. }\end{array}$ & $\begin{array}{l}\text { Unclear risk } \\
\text { Blinding unclear }\end{array}$ & $\begin{array}{l}\text { Low risk } \\
\text { None lost to } \\
\text { follow-up }\end{array}$ & $\begin{array}{l}\text { Low risk } \\
\text { All outcomes } \\
\text { reported }\end{array}$ & $\begin{array}{c}\text { Unclear } \\
\text { risk }\end{array}$ \\
\hline
\end{tabular}

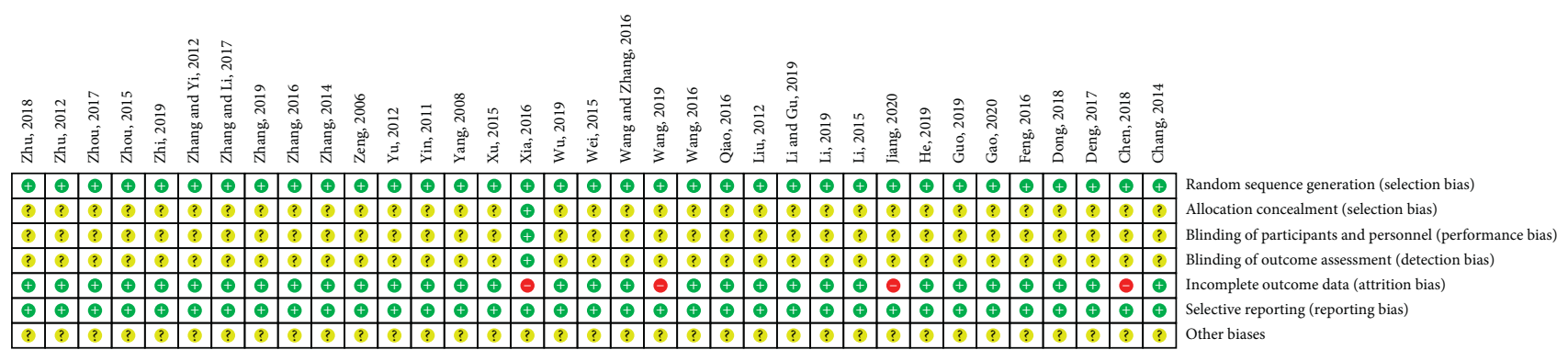

Figure 2: Potential risk of bias of each included study. 


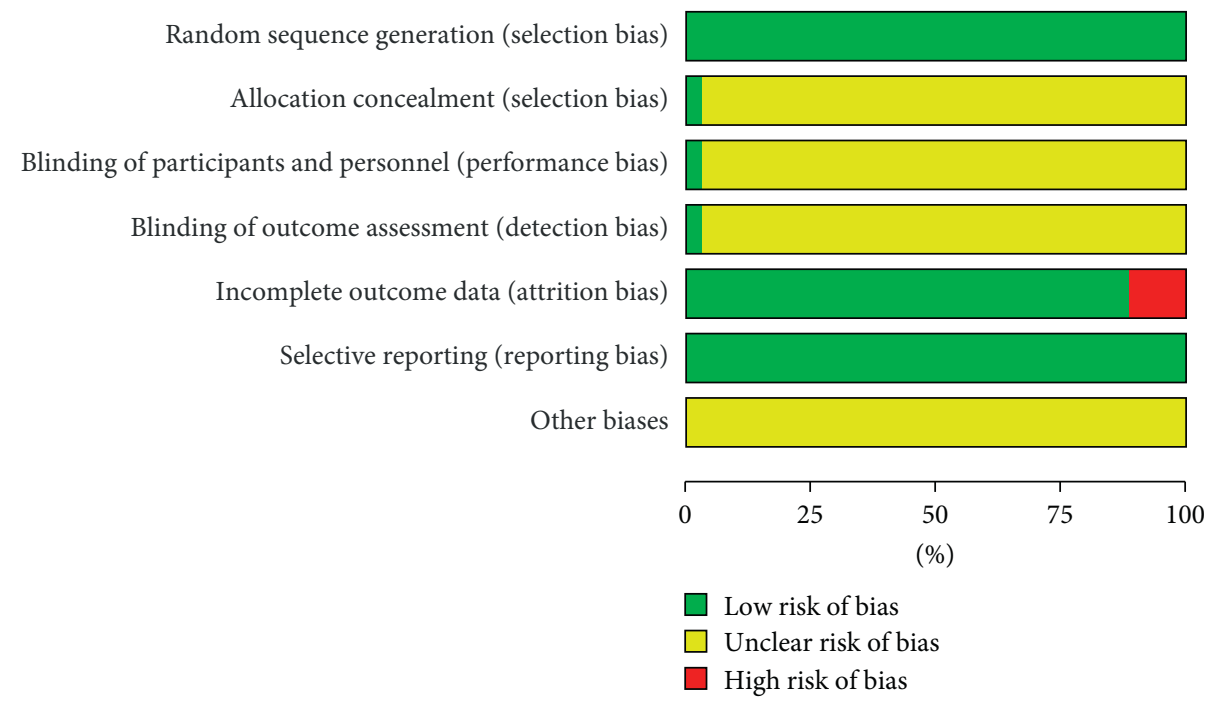

Figure 3: Summary of bias evaluation for the studies.

CI: 1.16 to $2.20, P<0.00001, I^{2}=91 \%$ ) (Figure 5). The heterogeneity was high, and we found that the treatment durations and the acupuncture methods were different in these 12 studies, so we performed a subgroup analysis. Subgroup analysis of six articles seemed to show that electroacupuncture combined with swallowing treatment was more effective than swallowing treatment alone $(\mathrm{MD}=1.75,95 \% \mathrm{CI}: 0.92$ to $2.58, P<0.00001)$ (Figure 6). The subgroup analysis of these six articles seemed to show that simple acupuncture combined with swallowing treatment was more effective than swallowing treatment alone $(\mathrm{MD}=1.62,95 \% \mathrm{CI}: 0.90$ to $2.34, P<0.00001)$ (Figure 6). However, we did not find a clear source of heterogeneity for IFRS with an $I^{2}$ statistic that ranged from $75 \%$ to $96 \%$ in subgroup analyses, such as different acupuncture stimulation parameters, different acupoint selections, different needle holding times, and treatment durations.

3.6. Videofluoroscopy(VFSS). Among the included studies, 8 used videofluoroscopy to evaluate the effectiveness of the treatment with continuous data. The result exhibited a MD with medium heterogeneity $\left(I^{2}=81 \%\right)$. We performed a subgroup analysis, and heterogeneity was found to remain unaltered although no source for it was identified. The metaanalysis showed that acupuncture combined with swallowing treatment produced a sustained and significant improvement, as reflected in the VFSS scores in these stroke patients $(\mathrm{MD}=2.26,95 \% \mathrm{CI}: 1.77$ to $2.74, P<0.00001)$ (Figure 7).

3.7. Watian Swallowing Test (WST). Eleven studies selected the Watian swallowing test as the evaluation standard. The meta-analysis showed a MD with high heterogeneity $\left(I^{2}=99 \%\right)$. We did not find a clear source of heterogeneity for WST with an $I^{2}$ statistic that ranged from $93 \%$ to $100 \%$ in subgroup analyses, such as different acupuncture stimulation parameters, different acupoint selections, different needle holding times, and treatment durations. We could see from the figure that the score of the control group was higher than that of the acupuncture group (Figure 8). This illustrated that the acupuncture group was able to lower the WST scores $(\mathrm{MD}=-1.21,95 \% \mathrm{CI}:-1.85$ to $-0.57, P=0.0002)$ (Figure 8).

3.8. Acupuncture Point. The selection of acupoints was chosen mainly based on the symptoms and syndrome differentiation of traditional Chinese medicine (TCM) (Figure 9). After analysis of points adopted in these trials, we found that Fengchi (GB20), Jinjin (EX-HN12), Yuye (EXHN13), Lianquan (RN23), and Yifeng (SJ17) were the five points most commonly used (Figure 10).

3.9. Publication Bias. Publication bias was reported via a funnel plot (Figure 11), in which the asymmetry of the funnel plots may have arisen through heterogeneity.

3.10. Adverse Events. Three studies reported adverse events $[28,32,49]$, while the remaining 32 studies did not mention adverse events. Among the 3 studies, they reported the occurrence of adverse events such as bleeding, pain, and discomfort. However, no life-threatening adverse events were noted in any of the included studies.

\section{Discussion}

The object of this systematic review was to evaluate the effectiveness of acupuncture in treating dysphagia after stroke. This systematic review showed that the therapeutic efficacy of acupuncture combined with other interventions was better than that of the control group in the SSA score, IFRS score, VFSS score, and WST score. In the subgroup analysis, we obtained similar results that acupuncture had a significant effect on dysphagia. 


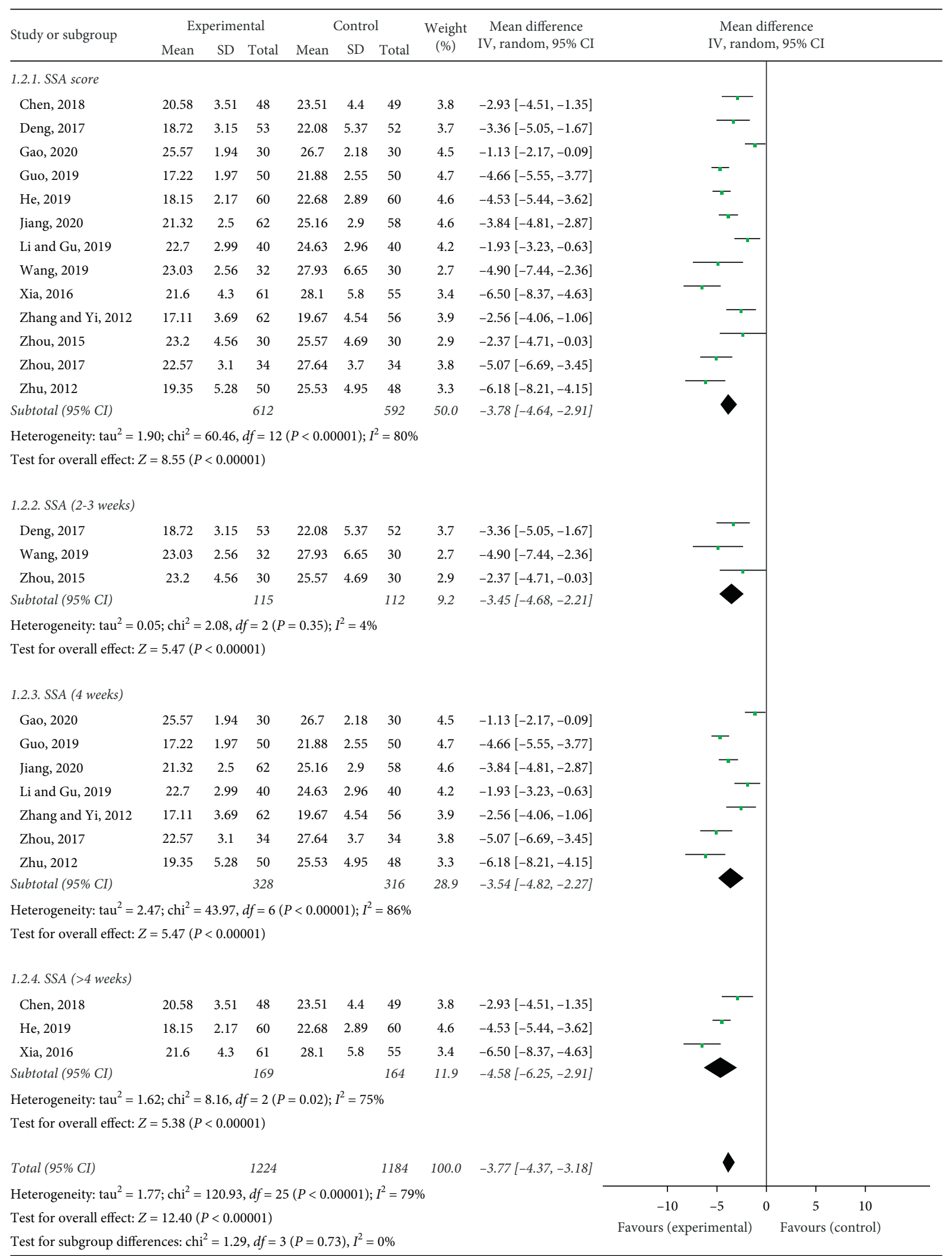

FIGURE 4: Forest plot of the SSA effective rate. 


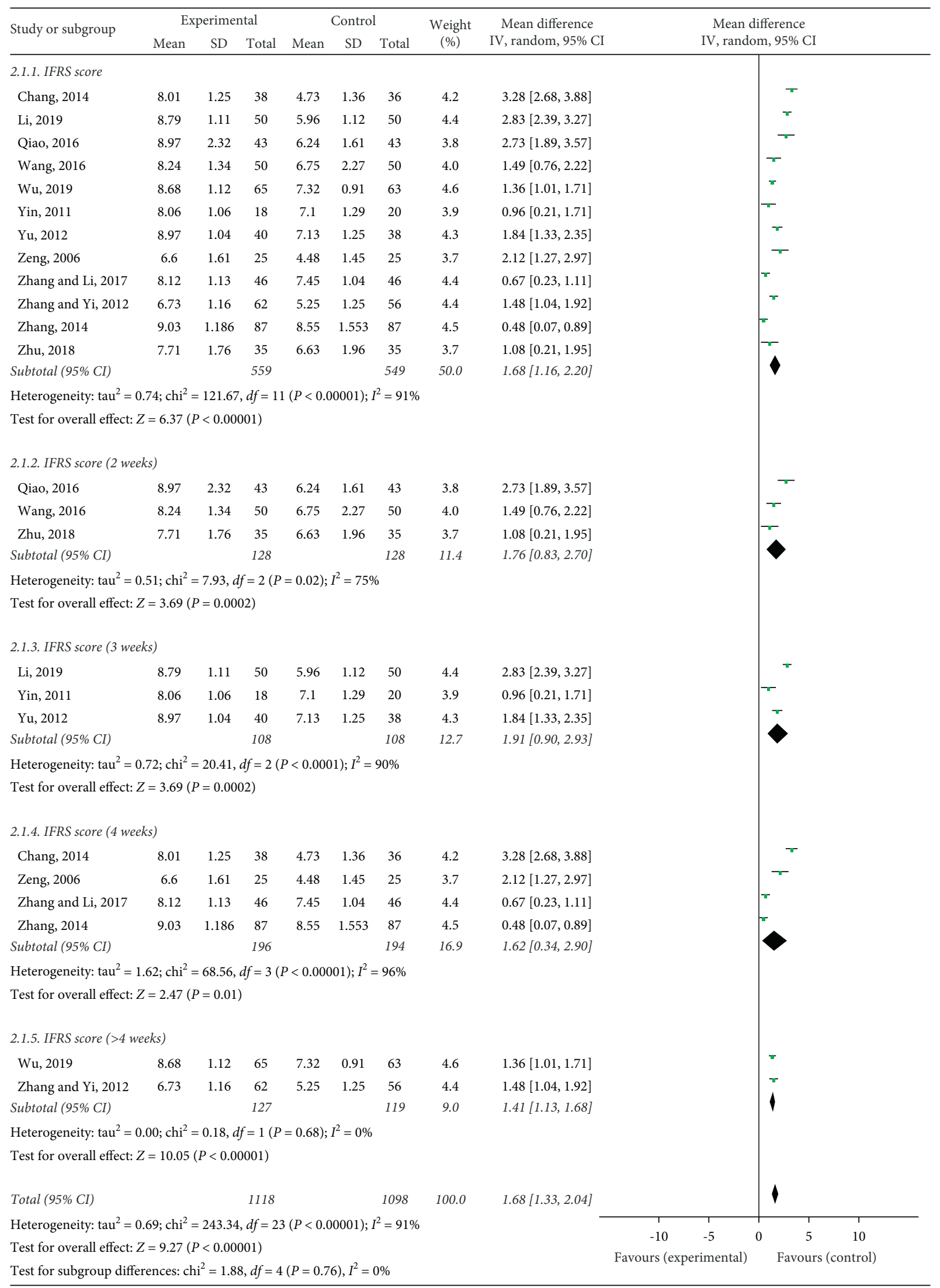

FIGURE 5: Forest plot of IFRS effective rate. 


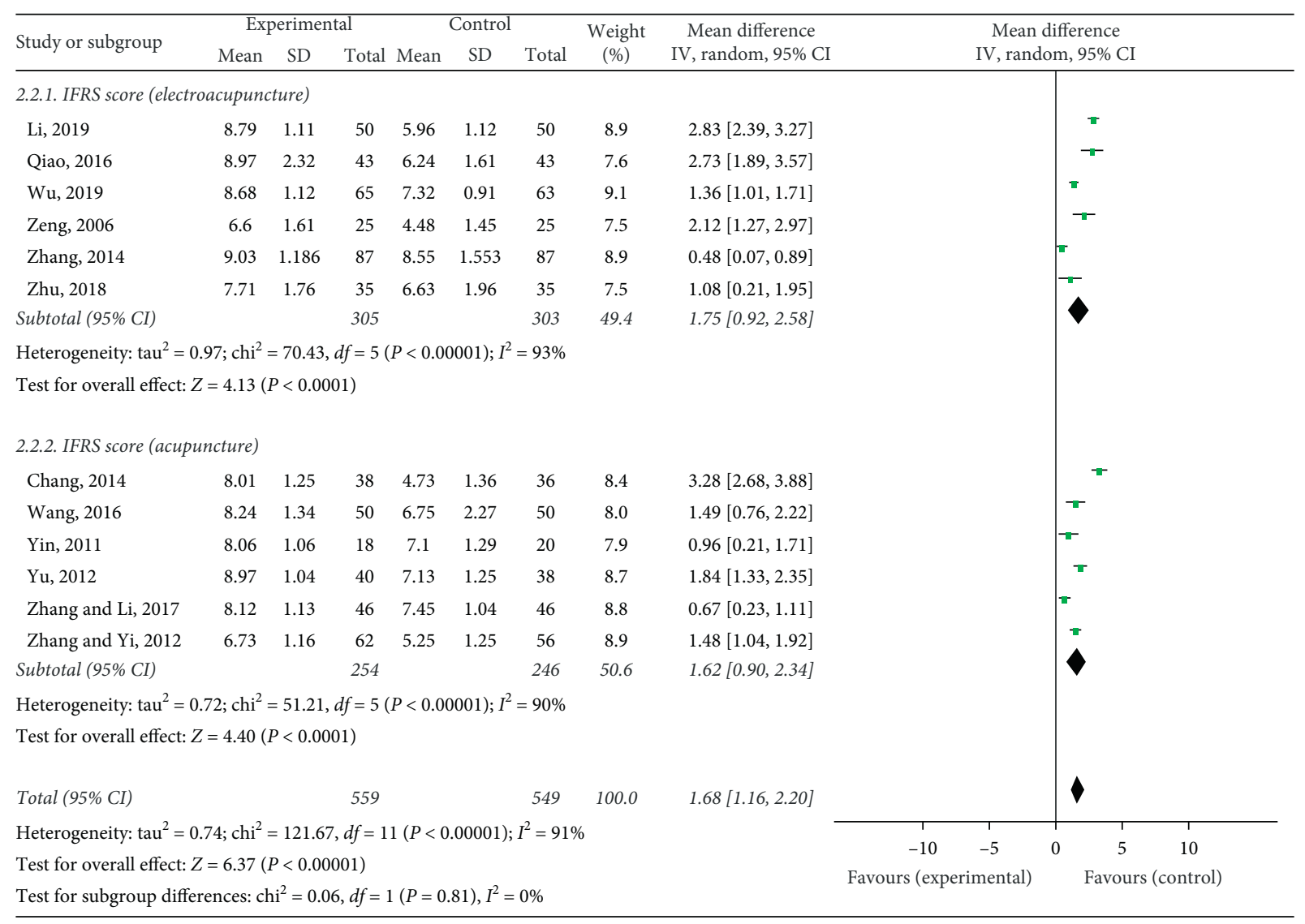

FIgURE 6: Forest plot of IFRS subgroup analysis.

The advantage of this systematic review was that most of the included RCTs had a low or moderate risk of bias. However, we acknowledge that there are some limitations in this review. First, a language bias may exist because all of the included trials were conducted and published by Chinese investigators. Second, in the present study, only one trial [28] reported allocation concealment, blinding of performance, and blinding of assessment; these risks of bias may affect the interpretation of the results. Finally, four trials $[25,28,31,40]$ excluded dropout participants for data analysis, which may increase the risk of attrition bias.

Poststroke dysphagia is in the category of "unsound speech and motor impairment" in TCM. It is believed in TCM that the etiology and pathogenesis of dysphagia are pathogenic wind, fire, phlegm, blood stasis, and qi deficiency, leading to the dysfunction of Zang-fu organs, reverse flow of qi and blood, obstruction of meridians and collaterals by blood stasis, and oppression of the brain marrow. The location of sickness is related to the brain, mouth, tongue, and throat. Hence, acupuncture can be used at the corresponding points to nourish yin, activate collaterals, wake up the brain, open the aperture, and remove obstruction. Some studies [59-61] showed that acupuncture therapy could improve the blood circulation in the cerebral cortex motor functional areas, promote the recovery of central nervous system function, improve the brain energy metabolism, activate the specific motor functional areas of the cerebral cortex, and promote the remodeling of brain function. This may be the main mechanism of acupuncture in treating dysphagia after stroke.

Meta-analyses showed that acupuncture could improve swallowing at different stages of treatment, and some studies $[28,34,55]$ showed that the longer the acupuncture treatment lasts, the better the recovery of swallowing function, which may be related to the time it takes to remodel brain functions. However, some studies [38, 48, 51] showed that the recovery of the swallowing function after acupuncture treatment for more than 4 weeks was not as good as that after acupuncture treatment for 4 weeks, which may be related to the different evaluation criteria of the efficacy of swallowing disorders and the diversity of acupuncture treatment options. VFSS and FEES are two instrumental assessments of dysphagia and are considered the "gold standard" for swallowing assessment [62, 63]. However, only 8 studies used VFSS to evaluate the swallowing performance of the participants, and most of the studies used the WST, SSA, and IFRS. These clinical evaluation scales were subjective clinical evaluation tools based on the observation of the evaluator, which may lead to inaccurate evaluation of the treatment effect. Moreover, in the therapeutic schedule of acupuncture, acupoint selection, stimulation method, needle holding time, and treatment durations in the included studies were not identical, which may affect the outcomes. Previous studies [64-66] showed that many factors influenced the 


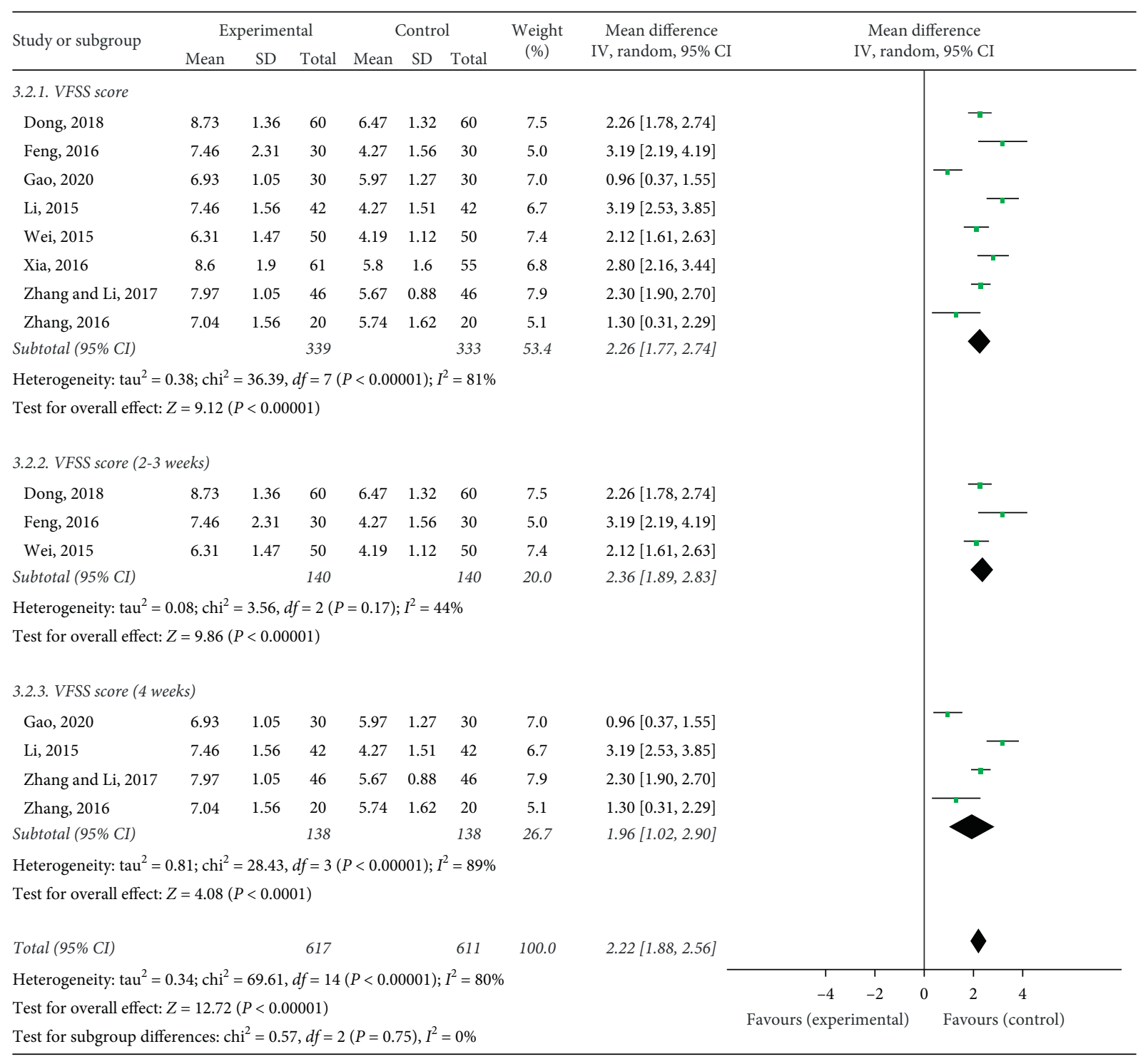

Figure 7: Forest plot of the VFSS effective rate.

efficacy of acupuncture, such as age, comorbidity, gender, disease severity, stimulation of acupuncture, expectations of patients, and doctor-patient interaction, which may be sources of heterogeneity. However, due to the inability to obtain more relevant data, we cannot analyze based on relevant influencing factors. It was necessary to use strict evaluation standards and high-quality RCT designs to explore acupuncture for dysphagia on poststroke.

Electroacupuncture is a technique of acupuncture based on a traditional acupuncture method combined with modern electrotherapy. Six of the 35 studies included in the meta-analysis used electroacupuncture. The results of subgroup analysis showed that both electroacupuncture and simple acupuncture could improve patients' swallowing function, but the effect was not significantly different between the two, which may be related to the small sample size, the diversity of acupuncture treatment options, and the different evaluation criteria for the efficacy of swallowing disorders. There is a need for more high-quality trials with large sample sizes to investigate electroacupuncture.

In this systematic review, the acupoints used in these 35 RCTs were different. Many studies employed individualized acupoints, but there were 5 points that were most commonly used. In this study, the acupoints of the nape were selected according to the adjacent therapeutic effects of the acupoints (Figure 9). Among them, Fengchi (GB 20), an important point for wind, can be used to suppress yang, extinguish wind, dissolve phlegm and benefit the throat, and clear away heat from the head [38]. Lianquan ( $\mathrm{RN} 23$ ) is an important acupoint mainly for aphasia and deglutition disorder, and it can be used to benefit the pharynx [28]. Jinjin (EX-HN12) and Yuye (EX-HN13) are acupoints for dredging meridians, activating collaterals, and regulating and smoothening qi and blood [55]. Yifeng (SJ17) can be used to open depression winds and benefit pharynges [32]. Hence, the above 5 acupoints could nourish yin, activate the collaterals, wake up 


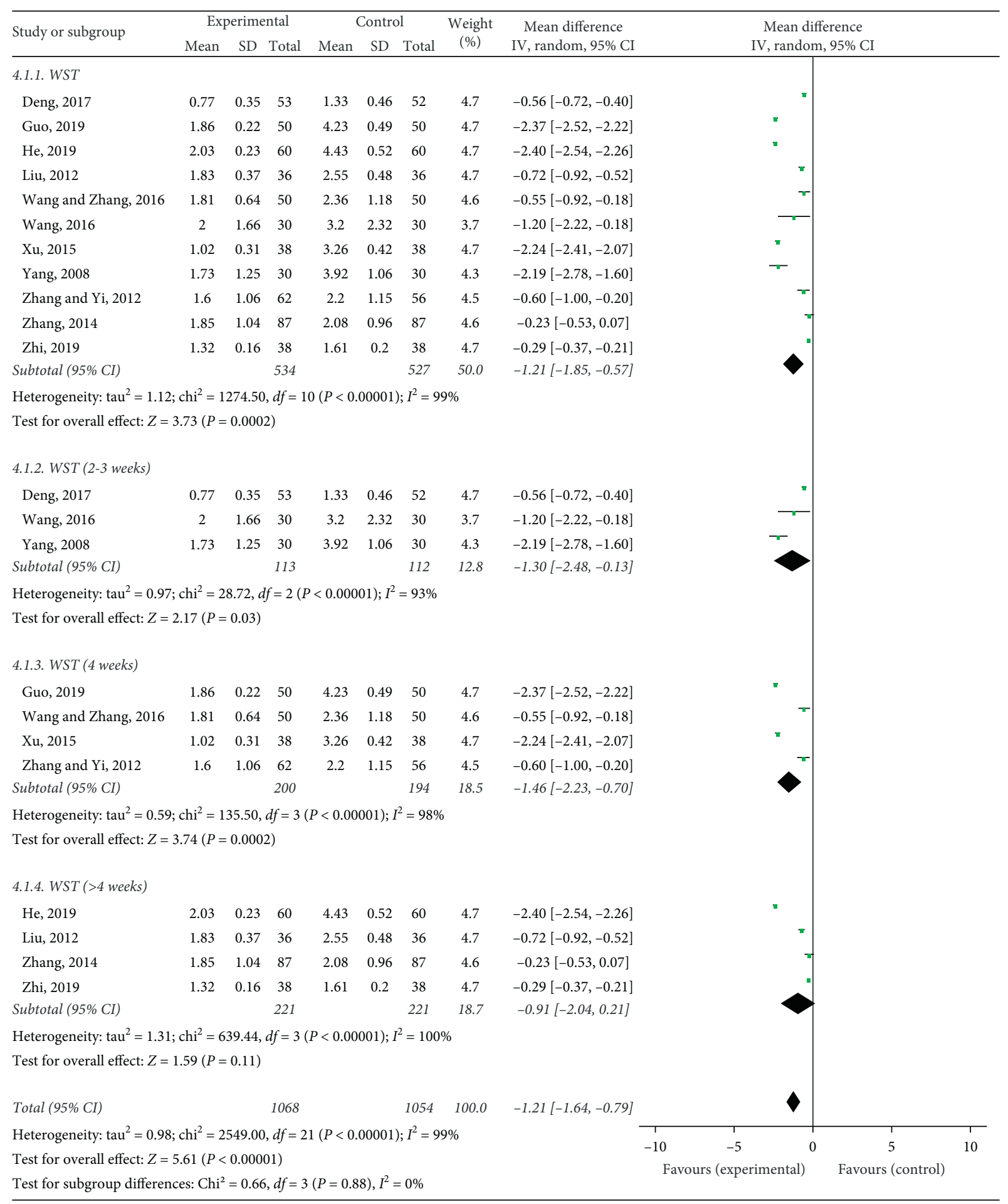

Figure 8: Forest plot of the WST effective rate.

the brain, open the aperture, and remove obstruction, thus benefiting dysphagia patients.

In this systematic review, only three studies [28, 32, 49] reported adverse events, including bleeding, pain, and discomfort, and the reactions were tolerable and not serious. The remaining RCTs did not mention any adverse events or side effects. Therefore, acupuncture is safe for dysphagia.
The results of this systematic review show that acupuncture may offer some benefits to patients with dysphagia. However, this review has several limitations. First, we searched only Chinese and English databases, which may cause publication bias. Second, most clinical evaluation scales included in this study were subjective clinical evaluation tools based on the observation of the evaluator, which 


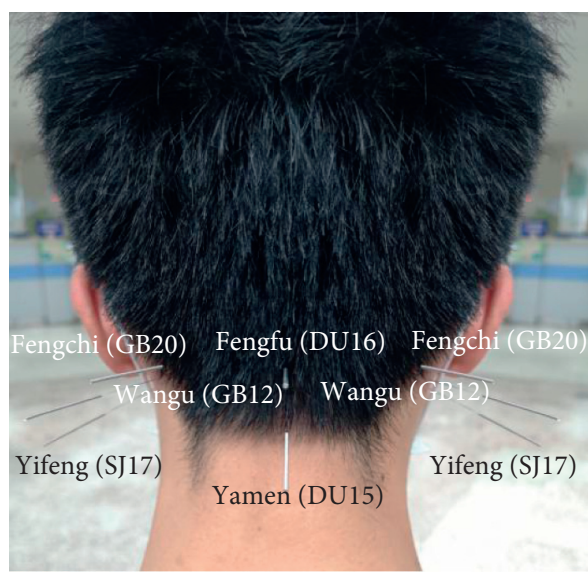

(a)

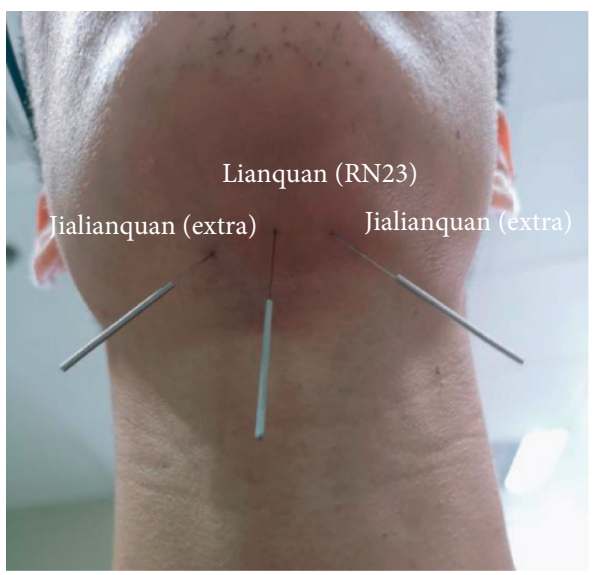

(b)

Figure 9: Acupoints in the neck: (a) bilateral Fengchi (GB20), bilateral Wangu (GB12), bilateral Yifeng (SJ17), Fengfu (DU16), and Yamen (DU15); (b) Lianquan (RN23) and bilateral Jialianquan (extra).

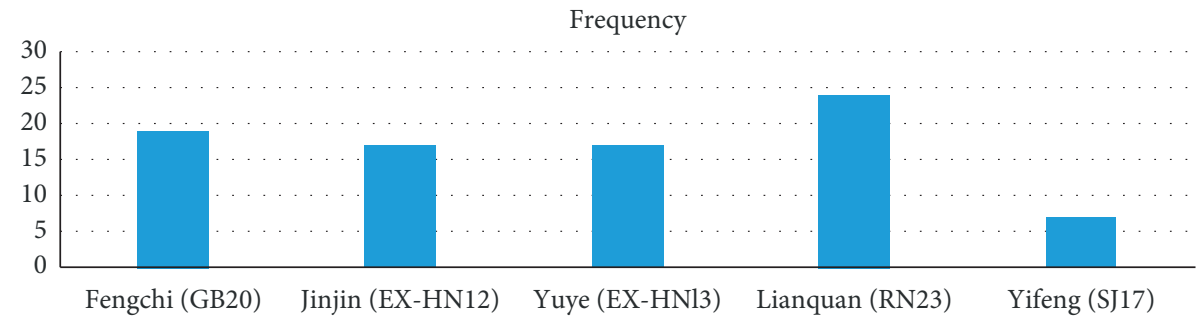

FIGURE 10: The most frequently used acupoints in these studies.

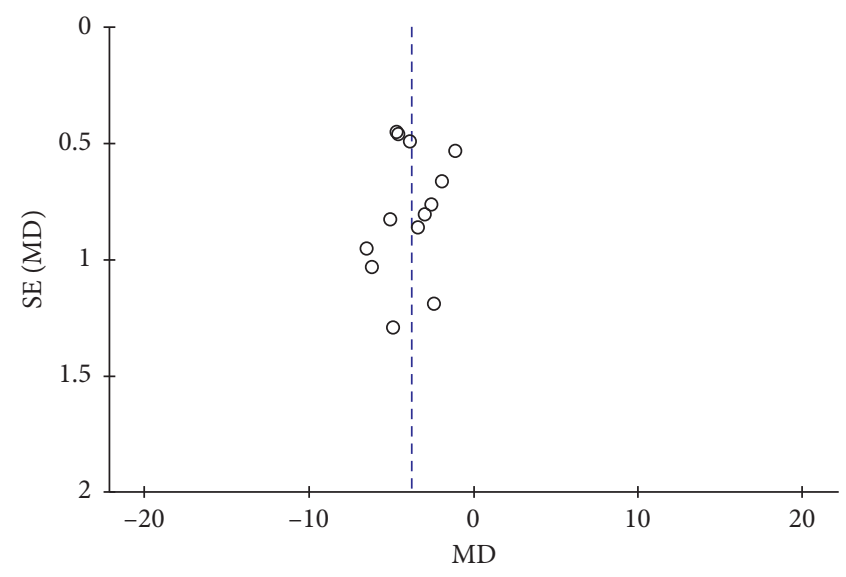

(a)

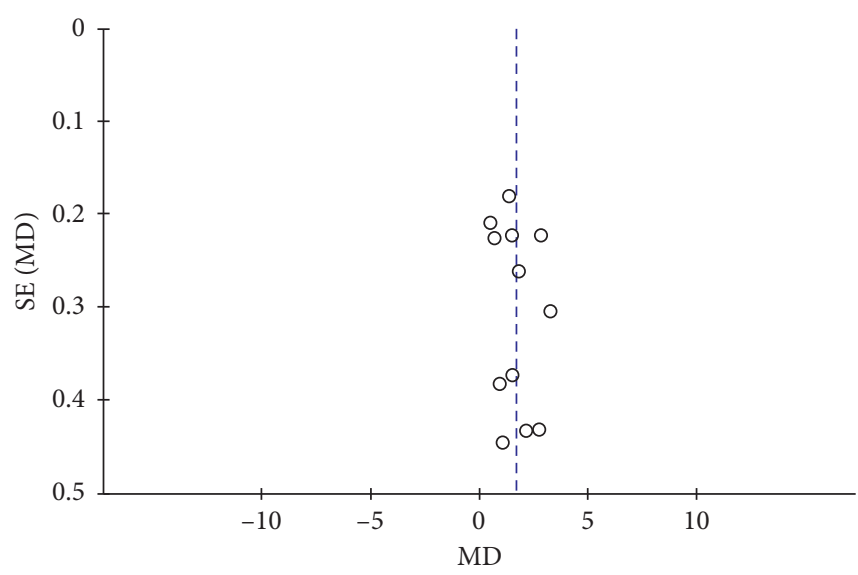

(b)

FIGURE 11: Continued. 


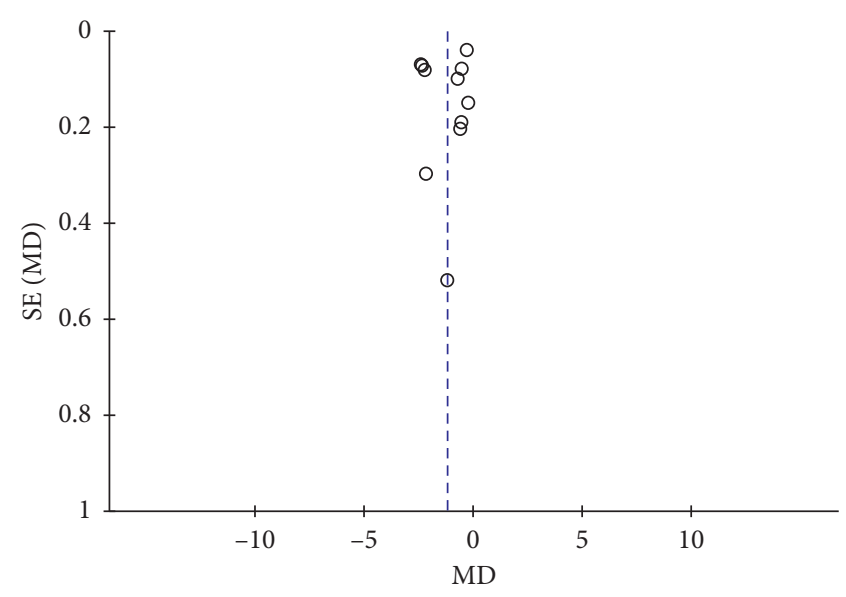

(c)

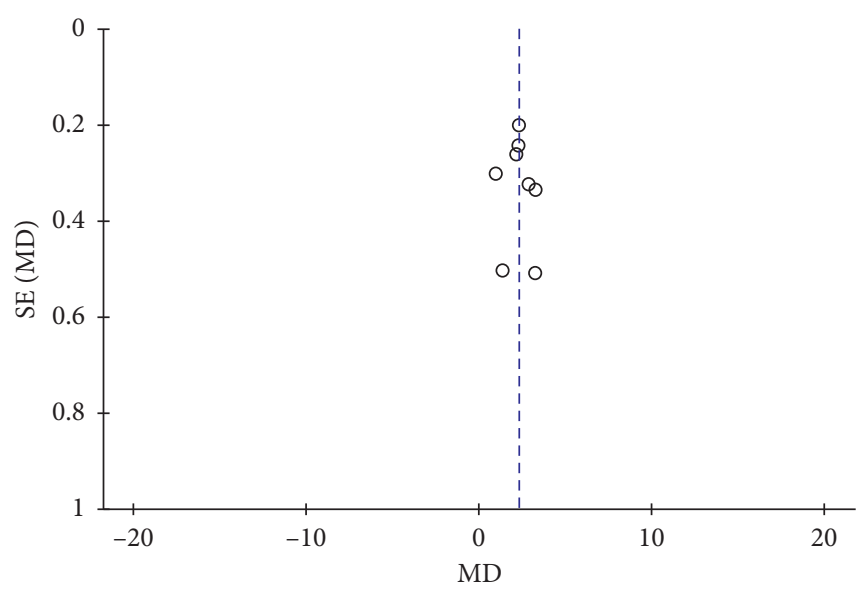

(d)

FIgURE 11: Funnel plot of the publication bias of acupuncture in the (a) SSA, (b) IFRS, (c) VFSS, and (d) WST.

may lead to an inaccurate evaluation of the treatment effect. Third, in the therapeutic schedule of acupuncture, acupoint selection, stimulation method, needle holding time, and treatment durations in the included studies were not identical, which may affect the outcomes. Fourth, in this systematic review, two studies [28, 51] reported a three-month follow-up, and the remaining RCTs reported only the short-term treatment, so the treatment duration and follow-up time were insufficient to draw conclusions. Fifth, the challenge of blinding arises from the unique nature of acupuncture treatment. Acupuncture treatment involves not only a device but also the acupuncture process and its techniques, such as needle insertion and needle manipulations. It was difficult to achieve true double blinding, which may cause a potential performance bias [67]. In the future, to minimize the ascertainment bias of subjects, implementation of the intervention should be carefully designed to achieve effective blinding of the subjects. The outcome assessor should be blinded to the treatment assignment to reduce detection bias in the study, and the statistician involved in data analysis is usually blinded to group assignments so that the data can be analyzed and interpreted appropriately without bias. These limitations could lead to highly heterogeneous results that prevent us from making a definitive conclusion.

\section{Conclusion}

In conclusion, acupuncture for dysphagia after stroke has therapeutic efficacy and safety. More strict evaluation standards and high-quality RCT designs are necessary for further exploring acupuncture for the treatment of dysphagia after stroke.

\section{Conflicts of Interest}

The authors declare that there are no conflicts of interest regarding the publication of this paper.

\section{Authors' Contributions}

All authors contributed to the writing and redrafting of the manuscript. Pu Wang had the original idea. Huiyu Liu and Xiao Bao provided support and advice. Lida Zhong, Jing Wang, and Fang Li performed literature search, assessed study quality, and undertook data collection. Results were analyzed by Lida Zhong. Results were interpreted and discussed by Lida Zhong and Jin Wang.

\section{Acknowledgments}

The authors thank Yuebei people's hospital and Sun Yat-sen University for providing the literature resources.

\section{References}

[1] L. Rofes, N. Vilardell, and P. Clavé, "Post-stroke dysphagia: progress at last," Neurogastroenterology \& Motility, vol. 25, no. 4, pp. 278-282, 2013.

[2] P. Clavé, L. Rofes, S. Carrión et al., "Pathophysiology, relevance and natural history of oropharyngeal dysphagia among older people," Stepping Stones to Living Well with Dysphagia, vol. 72, pp. 57-66, 2012.

[3] M. Arnold, K. Liesirova, A. Broeg-Morvay et al., "Dysphagia in acute stroke: incidence, burden and impact on clinical outcome," PLoS One, vol. 11, no. 2, Article ID e0148424, 2016.

[4] C. Geeganage, J. Beavan, S. Ellender et al., "Interventions for dysphagia and nutritional support in acute and subacute stroke," Cochrane Database of Systematic Reviews, vol. 10, Article ID CD000323, 2012.

[5] S. M. Lim, J. Yoo, E. Lee et al., "Acupuncture for spasticity after stroke: a systematic review and meta-analysis of randomized controlled trials," Evidence Based Complementary and Alternative Medicine, vol. 2015, Article ID 870398, 2015.

[6] Y. Hou, Y. Liu, M. Li et al., "Acupuncture plus rehabilitation for unilateral neglect after stroke: a systematic review and meta-analysis," Evidence Based Complementary and Alternative Medicine, vol. 2020, Article ID 5301568, 2020.

[7] S. Kwon, S. Y. Cho, S. U. Park et al., "Study on the evaluation of various types of sham acupuncture treatments in hemiplegic stroke patients: the protocol of a randomized clinical 
trial," Evidence Based Complementary and Alternative Medicine, vol. 2019, Article ID 7395927, 2019.

[8] L.-X. An, X. Chen, X.-J. Ren, and H.-F. Wu, "Electro-acupuncture decreases postoperative pain and improves recovery in patients undergoing a supratentorial craniotomy," The American Journal of Chinese Medicine, vol. 42, no. 5, pp. 1099-1109, 2014.

[9] S. Y. Seo, K.-B. Lee, J.-S. Shin et al., "Effectiveness of acupuncture and electroacupuncture for chronic neck pain: a systematic review and meta-analysis," The American Journal of Chinese Medicine, vol. 45, no. 8, pp. 1573-1595, 2017.

[10] Y. T. Chan, H. W. Zhang, W. Z. Sun et al., "Acupuncture for poststroke dysphagia: a pilot, nonrandomized, self-controlled trial," Evidence Based Complementary and Alternative Medicine, vol. 2020, Article ID 4689296, 2020.

[11] L. Chen, J. Fang, R. Ma et al., "Acupuncture for acute stroke: study protocol for a multicenter, randomized, controlled trial," Trials, vol. 15, no. 1, p. 214, 2014.

[12] P. M. Bath, H. S. Lee, and L. F. Everton, "Swallowing therapy for dysphagia in acute and subacute stroke," Cochrane Database of Systematic Reviews, vol. 10, no. 10, Article ID CD000323, 2018.

[13] D. Moher, A. Liberati, J. Tetzlaff, and D. G. Altman, "Preferred reporting items for systematic reviews and meta-analyses: the PRISMA statement," International Journal of Surgery, vol. 8, no. 5, pp. 336-341, 2010.

[14] D. M. Suiter and S. B. Leder, "Clinical utility of the 3-ounce water swallow test," Dysphagia, vol. 23, no. 3, pp. 244-250, 2008.

[15] J. Ellul and D. Barer, "On behalf of ESDB/COSTAR collaborative dysphagia study. Interobserver reliability of a standardised swallowing assessment (SSA)," Cerebrovascular Diseases, vol. 6, pp. 152-153, 1996.

[16] L. Perry, "Screening swallowing function of patients with acute stroke. part two: detailed evaluation of the tool used by nurses," Journal of Clinical Nursing, vol. 10, no. 4, pp. 474-481, 2001.

[17] L. Perry, "Screening swallowing function of patients with acute stroke. part one: identification, implementation and initial evaluation of a screening tool for use by nurses," Journal of Clinical Nursing, vol. 10, no. 4, pp. 463-473, 2001.

[18] J. C. Rosenbek, J. A. Robbins, E. B. Roecker, J. L. Coyle, and J. L. Wood, "A penetration-aspiration scale," Dysphagia, vol. 11, no. 2, pp. 93-98, 1996.

[19] M. A. Crary, G. D. C. Mann, and M. E. Groher, "Initial psychometric assessment of a functional oral intake scale for dysphagia in stroke patients," Archives of Physical Medicine and Rehabilitation, vol. 86, no. 8, pp. 1516-1520, 2005.

[20] B. Martin-Harris and B. Jones, "The videofluorographic swallowing study," Physical Medicine and Rehabilitation Clinics of North America, vol. 19, no. 4, pp. 769-785, 2008.

[21] C.-W. Kuo, C. T. Allen, C.-C. Huang, and C.-J. Lee, "Murray secretion scale and fiberoptic endoscopic evaluation of swallowing in predicting aspiration in dysphagic patients," European Archives of Oto-Rhino-Laryngology, vol. 274, no. 6, pp. 2513-2519, 2017.

[22] J. P. Higgins, D. G. Altman, P. C. Gøtzsche et al., "The cochrane collaboration's tool for assessing risk of bias in randomised trials," BMJ, vol. 343, Article ID d5928, 2011.

[23] J. P. T. Higgins, S. G. Thompson, J. J. Deeks et al., "Measuring inconsistency in meta-analyses," BMJ, vol. 327, no. 7414, pp. 557-560, 2003.

[24] Z. J. Wang, Y. Zhang, J. C. Qing et al., "Clinical study on acupuncture combined with swallowing training in the treatment of dysphagia after stroke," China Foreign Medical Treatment, vol. 35, no. 36, pp. 174-176, 2016.

[25] Y. J. Jiang, L. Lin, Y. M. You et al., "Clinical study on the treatment of swallowing dysfunction after stroke with three tongue needles," Shanghai Journal of Acupuncture and Moxibustion, vol. 39, no. 5, pp. 530-535, 2020.

[26] W.-b. Wu, D.-f. Fan, C. Zheng et al., "Relieving throat and opening orifice acupuncture therapy for the post-stroke dysphagia," World Journal of Acupuncture - Moxibustion, vol. 29, no. 1, pp. 37-41, 2019.

[27] H. T. Wei, T. Peng, D. Yang et al., "Clinical observation of acupuncture combined with rehabilitation training in the treatment of dysphagia after stroke," Journal of Emergency in Traditional Chinese Medicine, vol. 24, no. 10, pp. 1808-1810, 2015.

[28] W. G. Xia, C. J. Zheng, J. H. Xia et al., “Treatment of dysphagia after stroke by acupuncture based on syndrome differentiation: a randomized controlled study," Chinese Acupuncture \& Moxibustion, vol. 36, no. 7, pp. 673-678, 2016.

[29] H. H. Zeng, C. Ma, S. L. Wu et al., "Comparative observation on acupuncture treatment for dysphagia after stroke," Chinese Journal of Rehabilitation Medicine, vol. 21, no. 4, pp. 343-344, 2006.

[30] L. Chang, P. L. He, Z. Z. Zhou et al., "Observation on the effect of acupuncture combined with functional electrical stimulation on dysphagia after acute stroke," Chinese Acupuncture \& Moxibustion, vol. 34, no. 8, pp. 737-740, 2014.

[31] F. Y. Chen, X. P. Liu, Y. H. Bao et al., "Clinical observation on the treatment of pseudobulbar paralysis after stroke by "Xiang Zhen" combined with rehabilitation training," Shanghai Journal of Acupuncture and Moxibustion, vol. 37, no. 2, pp. 135-139, 2018.

[32] S. W. Feng, S. H. Cao, S. J. Du et al., "Acupuncture combined with swallowing training in the treatment of dysphagia after stroke: a randomized controlled study," Chinese Acupuncture \& Moxibustion, vol. 36, no. 4, pp. 347-350, 2016.

[33] T. T. Guo and X. H. Li, "Treatment of dysphagia after ischemic stroke with Xiang Wuzhen," China Journal of Chinese Medicine, vol. 34, no. 2, pp. 431-434, 2019.

[34] Q. S. He, L. F. Yang, and W. B. Wang, "Acupuncture treatment of dysphagia caused by ischemic stroke," China Journal of Chinese Medicine, vol. 34, no. 7, pp. 1551-1554, 2019.

[35] H. Y. Li and X. Y. Ma, " 42 cases of dysphagia after stroke treated with acupuncture," Henan Traditional Chinese Medicine, vol. 35, no. 2, pp. 289-290, 2015.

[36] J. Z. Li, J. H. Li, and Z. Y. Si, "Clinical observation of electroacupuncture on dysphagia after stroke," China's Naturopathy, vol. 27, no. 24, pp. 9-10, 2019.

[37] X. Z. Li, B. L. Gu, H. Zhou et al., "The effect of nape acupuncture on swallowing function in patients with cerebral infarction," Medical Journal of Chinese People's Liberation Army, vol. 44, no. 4, pp. 322-326, 2019.

[38] D.-D. Liu, X. Tong, J.-y. Kou, Y. Wei, T.-S. Yang, and L.-D. Qiao, "Influence of acupuncture on remodeling of swallowing functions for patients with pseudobulbar palsy after cerebral infarction," Journal of Acupuncture and Tuina Science, vol. 10, no. 1, pp. 44-48, 2012.

[39] Z. Q. Qiao, F. F. Chen, and Y. H. Luo, "43 cases of dysphagia after stroke treated with acupuncture," Henan Traditional Chinese Medicine, vol. 36, no. 8, pp. 1356-1358, 2016.

[40] Q. L. Wang and L. Chen, "Observation on the therapeutic effect of three tongue needles combined with VitalStim electric stimulation on dysphagia after stroke," Shanghai 
Journal of Acupuncture and Moxibustion, vol. 38, no. 2, pp. 183-187, 2019.

[41] Y. D. Wang, "Clinical observation on acupuncture treatment of dysphagia after stroke," Chinese Journal of Ethnomedicine and Ethnopharmacy, vol. 25, no. 23, pp. 89-90, 2016.

[42] B. Xu, "Analysis of therapeutic effect of acupuncture on stroke pseudobulbar palsy," Shanxi Journal of Traditional Chinese Medicine, vol. 36, no. 10, pp. 1413-1414, 2015.

[43] X. H. Yang and S. L. Zhang, "30 cases of dysphagia due to pseudobulbar palsy after stroke treated by acupuncture," Journal of Changchun University of Traditional Chinese Medicine, vol. 24, no. 6, p. 712, 2008.

[44] Z. H. Yu and J. F. Hu, "Observation on the effect of acupuncture combined with rehabilitation training on dysphagia after stroke," Shanghai Journal of Acupuncture and Moxibustion, vol. 31, no. 9, pp. 642-644, 2012.

[45] Y. H. Zhu, "Clinical observation on 50 cases of dysphagia after acute cerebral infarction treated by acupuncture," Jiangsu Journal of Acupuncture and Moxibustion, vol. 44, no. 7, pp. 64-65, 2012.

[46] T. X. Zhou, L. J. Li, E. H. Feng et al., "Clinical observation on the treatment of dysphagia after stroke with scalp acupuncture and swallowing speech diagnosis and treatment instrument," Journal of Guangzhou University of Traditional Chinese Medicine, vol. 34, no. 6, pp. 859-863, 2017.

[47] K. X. Zhou, H. N. Ou, D. Zheng et al., "Observation on the clinical effect of swallowing training under scalp acupuncture on dysphagia after stroke," Chinese Journal of Physical Medicine and Rehabilitation, vol. 37, no. 12, pp. 936-939, 2015.

[48] J. M. Zhi, X. H. Bu, G. Liu et al., "Effect of tongue acupuncture combined with rehabilitation training on swallowing function and limb function of patients with stroke in recovery period," Journal of Clinical Acupuncture and Moxibustion, vol. 35, no. 9, pp. 12-16, 2019.

[49] Y. Zhang and J. Li, "Therapeutic effect of acupuncture combined with VitalStim electric stimulation on dysphagia after ischemic stroke," Chinese Journal of Integrated Traditional and Western Medicine in Intensive and Critical Care, vol. 24, no. 4, pp. 346-350, 2017.

[50] X. L. Zhang, Z. M. Tang, L. Y. Mao et al., "Effect of acupuncture combined with repetitive transcranial magnetic stimulation on oral dysphagia after stroke," Chinese Journal of Physical Medicine and Rehabilitation, vol. 41, no. 4, pp. 257-260, 2019.

[51] S. Zhang and G. M. Zhang, "87 cases of dysphagia after stroke treated by acupuncture combined with swallowing rehabilitation training," Journal of Anhui University of Traditional Chinese Medicine, vol. 33, no. 5, pp. 56-59, 2014.

[52] R. Y. Zhang and Y. D. Yin, "Observation on therapeutic effect of kidney tonifying acupuncture on dysphagia after cerebral infarction," Shanghai Journal of Acupuncture and Moxibustion, vol. 31, no. 9, pp. 637-639, 2012.

[53] Q. Zhang, H. Y. Fu, H. Liu et al., "Effect of acupuncture combined with neuromuscular electrical stimulation on dysphagia after stroke," Chinese Journal of Rehabilitation, vol. 31, no. 1, pp. 39-41, 2016.

[54] Z. L. Yin, Z. X. Meng, Y. J. Xue et al., "Observation on the effect of swallowing training combined with acupuncture and electrical stimulation on moderate and severe dysphagia in the recovery period of stroke," Chinese Journal of Physical Medicine and Rehabilitation, vol. 33, no. 12, pp. 916-919, 2011.

[55] J. X. Gao and H. F. Zhou, "Observation on therapeutic effect of nape cluster acupuncture combined with swallowing function training on dysphagia in stroke," Chinese Acupuncture \& Moxibustion, vol. 40, no. 6, pp. 586-590, 2020.

[56] G. Q. Dong, "Observation on the therapeutic effect of acupuncture on 120 patients with dysphagia after stroke," The Journal of Medical Theory and Practice, vol. 31, no. 5, pp. 666-667, 2018.

[57] X. X. Deng and C. Wu, "Clinical study of Xingnaokaiqiao acupuncture combined with VitalStim swallowing therapeutic apparatus in the treatment of dysphagia in stroke," China Journal of Chinese Medicine, vol. 32, no. 226, pp. 466-469, 2017.

[58] P. Y. Zhu, D. Wu, D. Chen et al., "Treatment of dysphagia after cerebral hemorrhage with Yu's nape needle," Journal of Changchun University of Traditional Chinese Medicine, vol. 34, no. 2, pp. 282-284, 2018.

[59] L. F. Chen, J. Q. Fang, L. N. Chen et al., "The results and Enlightenment of modern acupuncture treatment for stroke based on neuroanatomy," Acupuncture Research, vol. 39, no. 2, pp. 164-168, 2014.

[60] Z. Xie, F. Cui, Y. Zou et al., "Acupuncture enhances effective connectivity between cerebellum and primary sensorimotor cortex in patients with stable recovery stroke," Evidence Based Complementary and Alternative Medicine, vol. 2014, Article ID 603909, 2014.

[61] N. Zhou and H. F. Zhou, "Clinical research progress of acupuncture in the treatment of dysphagia after stroke," Journal of Liaoning University of Traditional Chinese Medicine, vol. 16, no. 8, pp. 250-251, 2014.

[62] S. E. Langmore, "History of fiberoptic endoscopic evaluation of swallowing for evaluation and management of pharyngeal dysphagia: changes over the years," Dysphagia, vol. 32, no. 1, pp. 27-38, 2017.

[63] J. Allen, D. Blair, and A. Miles, "Assessment of videofluoroscopic swallow study findings before and after cricopharyngeal myotomy," Head \& Neck, vol. 39, no. 9, pp. 1869-1875, 2017.

[64] G. X. Shi, X. M. Yang, C. Z. Liu et al., "Factors contributing to therapeutic effects evaluated in acupuncture clinical trials," Trials, vol. 21, no. 13, p. 42, 2012.

[65] L. Klaus, N. Karin, A. Schneider et al., "How large are the nonspecific effects of acupuncture? A meta-analysis of randomized controlled trials," BMC Medicine, vol. 8, no. 1, p. 75, 2010.

[66] H. Pan, R. Jin, M. Li, Z. Liu, Q. Xie, and P. Wang, "The effectiveness of acupuncture for osteoporosis: a systematic review and meta-analysis," The American Journal of Chinese Medicine, vol. 46, no. 3, pp. 489-513, 2018.

[67] H. Chen, M. Yang, Z. Ning et al., "A guideline for randomized controlled trials of acupuncture," The American Journal of Chinese Medicine, vol. 47, no. 1, pp. 1-18, 2019. 